\title{
Hydrogen peroxide inducible clone-5 mediates reactive oxygen species signaling for hepatocellular carcinoma progression
}

\author{
Jia-Ru Wu${ }^{1}$, Chi-Tan Hu${ }^{3}$, Ren-In You ${ }^{2}$, Siou-Mei Pan ${ }^{3}$, Chuan-Chu Cheng ${ }^{2}$, Ming- \\ Che Lee ${ }^{4}$, Chao-Chuan Wu ${ }^{5}$, Yao-Jen Chang ${ }^{5}$, Shu-Chuan Lin ${ }^{3}$, Chang-Shan Chen ${ }^{2}$, \\ Teng-Yi Lin', Wen-Sheng Wu² \\ ${ }^{1}$ Institute of Medical Sciences, College of Medicine, Tzu Chi University, Hualien, Taiwan \\ ${ }^{2}$ Department of Laboratory Medicine and Biotechnology, College of Medicine, Tzu Chi University, Hualien, Taiwan \\ ${ }^{3}$ Research Centre for Hepatology, Department of Internal Medicine, Buddhist Tzu Chi General Hospital and Tzu Chi University, \\ Hualien, Taiwan \\ ${ }^{4}$ Department of Surgery, Buddhist Tzu Chi General Hospital, School of Medicine, Tzu Chi University, Hualien, Taiwan \\ ${ }^{5}$ Department of Surgery, Taipei Tzu Chi Hospital, Buddhist Tzu Chi Medical Foundation, School of Medicine, Tzu Chi University, \\ Hualien, Taiwan \\ ${ }^{6}$ Department of Laboratory Medicine, Hualien Tzu Chi Hospital, Buddhist Tzu Chi Medical Foundation, Hualien, Taiwan \\ Correspondence to: \\ Wen-Sheng Wu, e-mail: wuwstcu1234@yahoo.com.tw \\ Keywords: HGF, paxillin, metastasis, JNK, migration \\ Received: April 18, $2015 \quad$ Accepted: September 11, 2015 \\ Published: September 22, 2015
}

\section{ABSTRACT}

One of the signaling components involved in hepatocellular carcinoma (HCC) progression is the focal adhesion adaptor paxillin. Hydrogen peroxide inducible clone-5 (Hic-5), one of the paralogs of paxillin, exhibits many biological functions distinct from paxillin, but may cooperate with paxillin to trigger tumor progression. Screening of Hic-5 in 145 surgical HCCs demonstrated overexpression of Hic-5 correlated well with intra- and extra-hepatic metastasis. Hic-5 highly expressed in the patient derived HCCs with high motility such as HCC329 and HCC353 but not in the HCCs with low motility such as HCC340. Blockade of Hic-5 expression prevented constitutive migration of HCC329 and $\mathrm{HCC} 353$ and HGF-induced cell migration of HCC340. HCC329Hic-5(-), HCC353Hic-5(-), HCC372Hic-5(-), the HCCs stably depleted of Hic-5, exhibited reduced motility compared with each HCC expressing Scramble ShRNA. Moreover, intra/extrahepatic metastasis of HCC329Hic-5(-) in SCID mice greatly decreased compared with HCC329Scramble. On the other hand, ectopic Hic-5 expression in HCC340 promoted its progression. Constitutive and HGF-induced Hic-5 expression in HCCs were suppressed by the reactive oxygen species (ROS) scavengers catalase and dithiotheritol and c-Jun N-terminal kinase (JNK) inhibitor SP600125. On the contrary, depletion of Hic-5 blocked constitutive and HGF-induced ROS generation and JNK phosphorylation in HCCs. Also, ectopic expression of Hic-5 enhanced ROS generation and JNK phosphorylation. These highlighted that Hic-5 plays a central role in the positive feedback ROS-JNK signal cascade. Finally, the Chinese herbal derived anti-HCC peptide LZ-8 suppressed constitutive Hic-5 expression and JNK phosphorylation. In conclusion, Hic-5 mediates ROS-JNK signaling and may serve as a therapeutic target for prevention of HCC progression.

\section{INTRODUCTION}

Hepatocellular carcinoma (HCC) is one of the most common deadly cancers worldwide. The poor prognosis of HCC is due to high recurrence rate mainly caused by intrahepatic metastasis (about $80 \%$ ) or extrahepatic metastasis (about 20\%) [1]. Therefore, prevention of metastasis is essential for HCC management. To address the issue, the suitable targets within the molecular pathways leading to HCC metastasis are needed to be identified. 
The tumor microenvironment of HCC contains a lot of metastatic factors including transforming growth factor $\beta$ (TGF $\beta$ ) [2] and hepatocyte growth factor (HGF) $[3,4]$, which are capable of triggering HCC metastasis. Paxillin, one of the adaptor molecules critical for integrating the focal adhesion signaling [5-7], is known to be involved in HCC progression triggered by HGF $[8,9]$, integrin engagement [10] or overexpression of P21-activated protein kinase [11].

Within the paxillin superfamily, Hic-5 is the most homologous to paxillin, with minor differences in the number of N-terminal LD domains [5]. However, many biochemical properties, regulatory mechanisms and molecular functions of Hic-5 are rather different from those of paxillin [12]. Upon integrin engagement or growth factor stimulation, paxillin becomes phosphorylated, primarily on Tyr 31 and 118 [5, 6] or Ser 178 [13], mediating signal transductions for cell spreading and motility. Although there are tyrosine phosphorylation sites on Hic-5 such as Y38, Y60 between LD1 and LD2 domain [for review 12, 33], the aforementioned phosphorylating events of paxillin (at Y31, 118) do not occur on Hic-5, due to the lack of cognate tyrosine/Ser residues. On the other hand, expression of Hic5 but not paxillin can be stimulated by TGF $\beta$ [14] or ROS [15], required for its biological activation. In spite of these discrepancies, Hic-5 was also capable of triggering tumor progression as paxillin, although via distinct molecular pathways [16]. Moreover, Hic-5 may cooperate with paxillin to regulate metastasis of breast cancer [17]. However, the role of Hic-5 in HCC has not been clarified thus far.

In this study, we found Hic-5 could be a potential prognosis maker and therapeutic target for prevention of HCC. On the signaling level, Hic-5 mediates the sustained ROS-JNK signaling required for triggering HCC progression.

\section{RESULTS}

\section{Detection of Hic-5 as a HCC progression marker}

Initially, we investigated whether Tyr31 phosphorylated paxillin [PXN (p-Y31)] can be a marker of HCC progression by examining the status of PXN(p-Y31) in HCC tissues obtained from surgery in TUZ CHI Hospital. In the pilot study using Western blot analysis, about $40.7 \%$ of the HCCs exhibited significant elevation of PXN(p-Y31) in tumor tissues, compared with that in the normal counterpart (Supplementary Figure S1, upper panel). Surprisingly, one more intensive band (around $50 \mathrm{kD}$ ) beneath PXN(p-Y31) (63-68 kD) can be observed in most of the HCCs screened as positive (Supplementary Figure S1, upper panel). We suspected this to be a protein belonging to the paxillin superfamily that strongly cross-reacted with the $\mathrm{Ab}$ against PXN(p-Y31). According to a previous report [5], Hic-5, one of the paxillin paralog with molecular weight most close to $50 \mathrm{kD}$, was suggested as the most possible candidate. Strikingly, Western blot of Hic-5 demonstrated dramatic elevation of Hic-5 in most of the HCCs with positive PXN(p-Y31) (Supplementary Figure S1, lower panel). To ascertain that the cross-reacted band was indeed Hic-5 but not other proteins related to paxillin, we further screened the expression of Hic-5 compared with leupaxin, another member of paxilin family with molecular weight slightly lower than $50 \mathrm{kD}$ [5], in more HCC samples. As demonstrated in the Western blot of Hic-5 (Figure 1A upper panel), overexpression of Hic-5 (50 kD) can be observed in 6 HCCs but not the normal counterparts, whereas no band can be clearly observed in the same HCCs by Western blot of leupaxin (Figure 1A lower panel). Further, by screening 145 HCCs we found PXN(p-Y31) and Hic-5 increased in about $40.7 \%$ and $48.3 \%$ tissues respectively. Moreover, about $34.5 \%$ HCC tissues exhibited simultaneous elevation of both proteins (Supplementary Figure S2). Whether Hic-5 indeed expressed within the HCCs was examined in situ. As demonstrated in Figure 1B, IHC of Hic-5 on tissue sections of two Hic-5 overexpressing HCCs, denoted as HCC-Hic-5 I and HCC-Hic-5 II, revealed that Hic-5 was distributed within the tumor (but not non-tumor) region as indicated by hematoxylin/Eosin (H.E.) stain on the parallel tissue sections. In contrast, IHC using IgG as control revealed negative staining in the same region.

Further, the correlations of increased expression of Hic-5 and PXN(p-Y31) with metastatic potential of HCCs were analyzed. As shown in Table 1, 83.3\%, 100\% and $84.2 \%$ of HCCs with positive intrahepatic metastasis (I.M.), extrahepatic metastasis (E.M.) and total metastasis (T.M.), respectively, exhibited higher expression of Hic-5. In contrast, Hic-5 was higher in only $43.3 \%$, 46\% and $42.9 \%$ of HCCs with negative I.M., E.M. and T.M, respectively. Statistical analysis revealed that high Hic-5 expression in HCCs correlated well with I.M. $(p=0.001)$, E.M. $(p=0.01)$ and T.M. $(p=0.001)$ (Chi-square test, SPSS 16.0, $N=145$ ) (Table 1). Also, the association of high Hic-5 with E.M. was confirmed by Fisher's Exact test $(p=0.011)$ (Table 1). In comparison, the correlations of PXN(p-Y31) with I.M. $(p=0.004)$ and T.M. $(p=0.008)$ were slightly poor than those of Hic-5 (Chi-square test, SPSS 16.0, $N=145$ ) (Table 2). In addition, the correlations of PXN(p-Y31) with E.M. was insignificant ( $p=0.186)$.

\section{Validating the role of Hic-5 in HCC progression using patient derived cell lines}

We further focused on investigating whether Hic-5 plays an essential role in the progression of HCC. Initially, we compared the expression of Hic-5 in several patientderived HCC cell lines, the phenotypes of which such as cell motility have been characterized [20]. As shown in Figure 2A, Hic-5 markedly expressed in most of the HCCs such as HCC329, HCC353, HCC365 and HCC372 with average to high motility, slightly expressed in HCC363 with average motility but not in the non-motile HCC 340 and HCC374 [20]. Notably, HCC329, the most motile 
(A)

\section{Case Number}

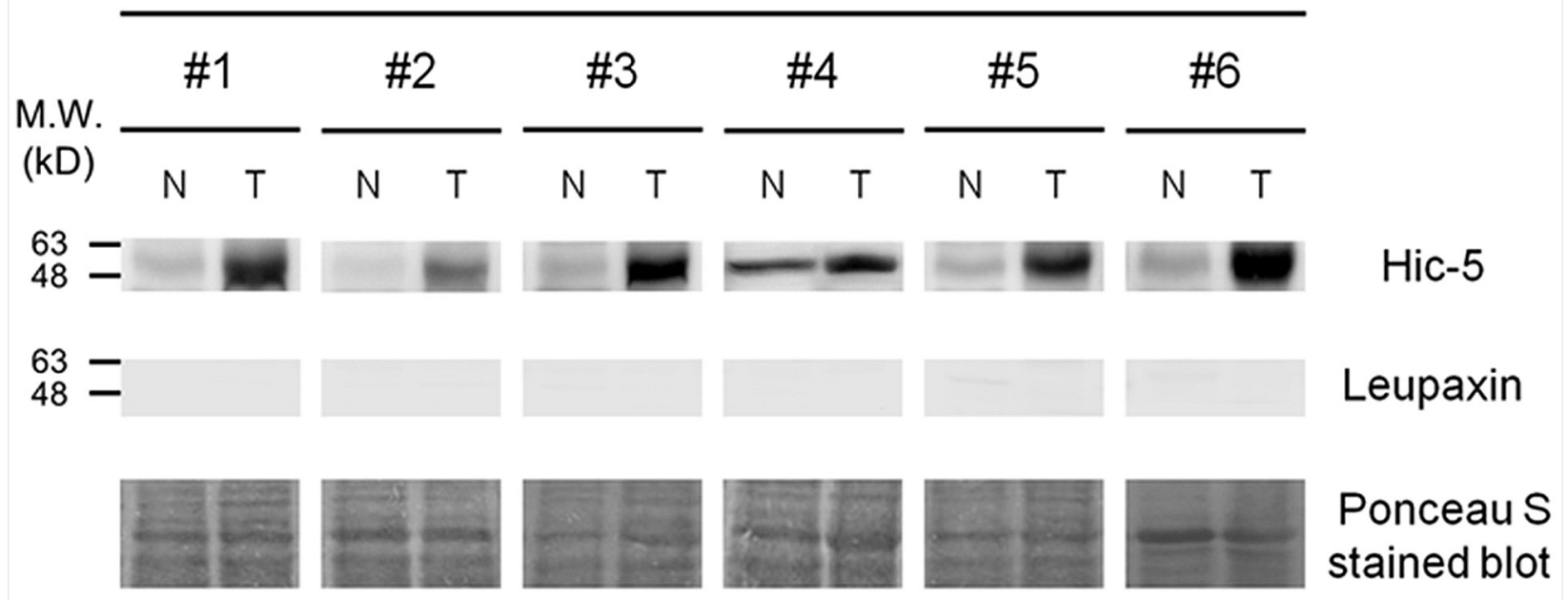

(B)

HCC-Hic-5 I
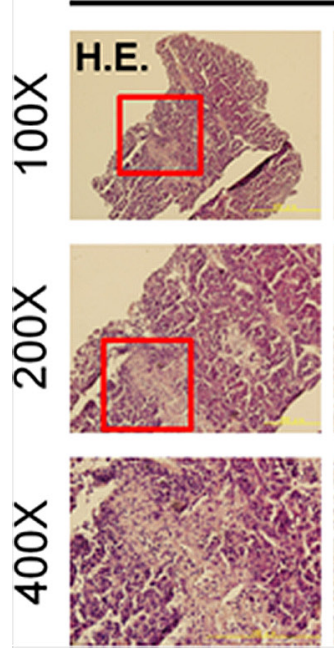
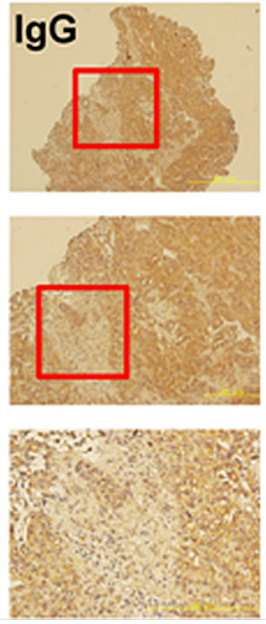
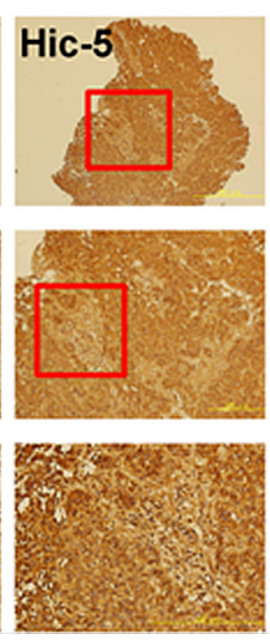

HCC-Hic-5 II
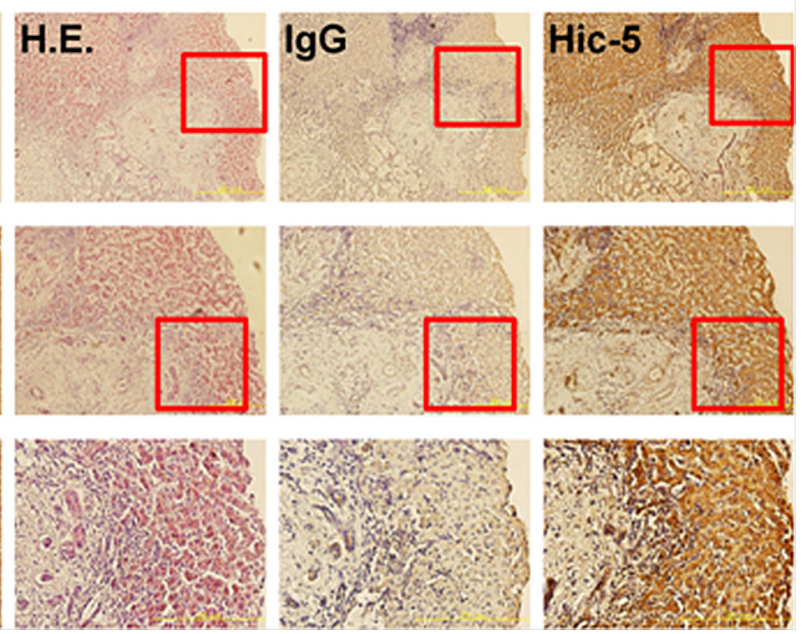

Figure 1: Detection of Hic-5 and Tyr31-phosphorylated paxillin in HCC tissues. (A) Western blot of Hic-5 (upper panel) and leupaxin (middle panel) in tissue lysates of HCC from indicated patients using ponceau S stain as loading control. The data was representative of 3 reproducible experiments. N and T represent non-tumor and tumor sample respectively. The locations of indicated molecular weight marker are shown on the left. (B) IHC of Hic-5 coupled with IgG negative control and H \& E stain were performed on the parallel tissue sections of 2 HCC cases. Red rectangle indicated the area within 100X field magnified to $200 \mathrm{X}$ and $400 \mathrm{X}$ shown below. The dark brown staining in Hic- 5 IHC revealed the location of indicated molecule in contrast with the light brown staining of the same area in the negative IgG control.

HCC [20], expressed the highest Hic-5 among the HCCs examined.

We further investigated whether Hic-5 can be induced in the low Hic-5 expressing HCCs such as HCC $340, \mathrm{H} 363$ and HCC374 by metastatic factors such as HGF and the tumor promoter 12-O-tetradecanoylphorbol-13-acetate (TPA), known to be a potent inducer of HCC migration [19, 21, 22]. HepG2, the conventionally used HCC cell line with low motility and no constitutive Hic-5 expression as HCC340 (data not shown), was also 
Table 1: Statistic analysis of the correlation of Hic-5 expression with $\mathrm{m}$ Metastatic potentials of HCC

\begin{tabular}{|c|c|c|c|c|}
\hline \multirow[t]{2}{*}{ Metastatic potentials } & \multicolumn{3}{|c|}{ Hic-5 } & \multirow[t]{2}{*}{$P$ value* } \\
\hline & All cases & $\begin{array}{c}\text { Low } \\
\text { expression }(T<N)^{\#}\end{array}$ & $\begin{array}{c}\text { High } \\
\text { expression }(T>N)^{\&}\end{array}$ & \\
\hline \multicolumn{5}{|c|}{ Intra-hepatic Metastasis $^{a}$} \\
\hline Positive & 18 & $3(16.7 \%)^{d}$ & $15(83.3 \%)$ & \multirow{2}{*}{0.001} \\
\hline Negative & 127 & $72(56.7 \%)$ & $55(43.3 \%)$ & \\
\hline \multicolumn{5}{|c|}{ Extra-hepatic Metastasis ${ }^{b}$} \\
\hline Positive & 6 & $0(0 \%)$ & $6(100 \%)$ & \multirow{2}{*}{$0.010(0.011 \%)$} \\
\hline Negative & 139 & $75(54.0 \%)$ & $64(46.0 \%)$ & \\
\hline \multicolumn{5}{|l|}{ Total Metastasis ${ }^{\mathrm{c}}$} \\
\hline Positive & 19 & $3(15.8 \%)$ & $16(84.2 \%)$ & \multirow{2}{*}{0.001} \\
\hline Negative & 126 & $72(57.1 \%)$ & $54(42.9 \%)$ & \\
\hline
\end{tabular}

"Hic-5 expression in HCC tissue (T) lower than that in the normal counterpart (N) by over 1.5-2.0 fold (Note: the quantitation of Hic-5 expression in Table $1 \& 2$ were performed based on results of Western blot of Hic-5 in HCC tissues).

${ }^{\&}$ Hic-5 expression in HCC tissue (T) higher than that in the normal counterpart (N) by over 1.5-2.0 fold.

*Statistical significance between high Hic-5 with indicated metastatic potential estimated by Chi-square test

${ }^{\%}$ Statistical significance between high Hic-5 with indicated metastatic potential estimated by Fisher' Exact test

${ }^{a}$ Sites of intra-hepatic metastasis (I.M.) coupled with vascular invasion in liver, including microscopic vascular invasion in small vessels and macroscopic tumor invasion in bile duct, hepatic vein and portal vein can be observed.

${ }^{b}$ Extra-hepatic metastasis (E.M.) including regional lymph node metastasis and distant organ metastasis can be observed.

${ }^{\mathrm{c}}$ Total Metastasis is equal to the summation of I.M. and E.M.

${ }^{\mathrm{d}}$ The percentage in parenthesis represent the ratio of the number of HCCs with indicated Hic-5 expression status (i.e. $T<N$ or $T>N$ ) vs total number of HCCs (all case) classified as either positive or negative of the indicated metastatic potential.

included. As shown in Figure 2B, Hic-5 can be induced in HCC 340 by $25 \mathrm{nM} \mathrm{HGF}$ to 3.8 -fold at $16 \mathrm{~h}$ and increased to 8.0 -fold at $24 \mathrm{~h}$. Also, $50 \mathrm{nM}$ TPA can induce Hic-5 expression to 4.0 -fold at $16 \mathrm{~h}$, which sustained until $24 \mathrm{~h}$ in HCC340. Consitently, both HGF and TPA can induce cell migration of HCC340 to 2.0 and 2.7-fold, respectively (Figure 2C). Similarly, HGF induced migration of HCC363 and HepG2 to 3.8- and 2.0-fold, respectively (Figure 5D), consistent with the HGF-induced Hic-5 expression in HepG2 and HCC363 (Supplementary Figure S3 and data not shown). However, HGF didn't induce Hic5 expression in $\mathrm{HCC} 374$ at $24 \mathrm{~h}$, although cell migration of HCC 374 can be induced by HGF at $48 \mathrm{~h}$ (data not shown).

Whether Hic-5 was required for constitutive and inducible cell migration of HCC were investigated by transient RNA interference. As demonstrated in Figure 3A, motility of HCC329 and HCC372 greatly decreased after transfection with Hic- 5 siRNA for 48 h by $60 \%$, compared with the control siRNA group. The efficiency of Hic-5 siRNA was verified by the reduction of Hic-5 (by 85-92\%) in both HCCs transfected with Hic-5 siRNA (Figure 3B). On the other hand, cell migration of HCC340 and HepG2 induced by HGF was decreased by prior transfection of the cells with Hic-5 siRNA by $73 \%$ and $100 \%$, respectively, compared with the control siRNA group (Figure 3C). The efficiency of Hic-5 siRNA was validated by that HGF-induced Hic-5 expression in HCC340 and HepG2 transfected with Hic-5 siRNA decreased by $84 \%$ (Figure 3D) and 99\% (Figure 7D), respectively, compared with the control siRNA group. To examine whether Hic-5 is sufficient for triggering cell migration of HCC, a Hic-5 cDNA plasmid, TGFB1I1, driven by the CMV promoter was employed. Remarkably, cell migration of HCC340, transfected with TGFB1I1 for $48 \mathrm{~h}$ increased to 3.0-fold, compared with the vector group (Figure $3 \mathrm{E}$ ). Figure $3 \mathrm{~F}$ demonstrated the dramatic increase of Hic-5 expression (to 7.5-fold) in cell transfected with TGFB1I1. Taken together, Hic-5 was essential for cell migration of HCCs.

To validate the role of Hic-5 in HCC progression, $\mathrm{HCC} 329$, HCC372 and HCC353 (the HCCs with high Hic-5) stably depleted of Hic-5, denoted as HCC329Hic-5(-), HCC372 Hic-5(-), and HCC353 Hic-5(-), respectively, were established by infection of the cells with a pseudoviral particle containing Hic-5 shRNA sequence inserted within a lentiviral vector. For comparison, each HCC was infected with the scramble shRNA to obtain HCC329Scramble, HCC372Scramble 
Table 2: Statistic analysis of the correlation of Tyr31- phosphorylated paxillin [PXN (p-Y31)] with Metastatic potentials of $\mathrm{HCC}$

\begin{tabular}{|c|c|c|c|c|}
\hline \multirow[t]{2}{*}{ Metastatic potentials } & \multicolumn{3}{|c|}{ PXN (p-Y31) } & \multirow[t]{2}{*}{$P$ value* } \\
\hline & All cases & $\begin{array}{c}\text { Low expression } \\
(T<N)\end{array}$ & $\begin{array}{c}\text { High expression } \\
\quad(T>N)\end{array}$ & \\
\hline \multicolumn{5}{|c|}{ Intra-hepatic Metastasis } \\
\hline Positive & 18 & $5(27.8 \%)$ & $13(72.2 \%)$ & \multirow{2}{*}{0.004} \\
\hline Negative & 127 & $81(63.8 \%)$ & $46(36.2 \%)$ & \\
\hline \multicolumn{5}{|c|}{ Extra-hepatic Metastasis } \\
\hline Positive & 6 & $2(33.3 \%)$ & $4(66.7 \%)$ & \multirow{2}{*}{0.186} \\
\hline Negative & 139 & $84(60.4 \%)$ & $55(39.6 \%)$ & \\
\hline \multicolumn{5}{|l|}{ Total Metastasis } \\
\hline Positive & 19 & $6(31.6 \%)$ & $13(68.4 \%)$ & \multirow{2}{*}{0.008} \\
\hline Negative & 126 & $80(63.5 \%)$ & $46(36.5 \%)$ & \\
\hline
\end{tabular}

\#PXN (p-Y31) in HCC tissue (T) lower than that in the normal counterpart $(\mathrm{N})$ by over 1.5-2.0 fold.

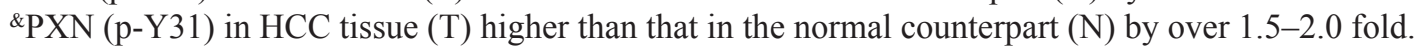

${ }^{*}$ Statistical significance between high PXN (p-Y31) with indicated metastatic potential estimated by Chi-square test

${ }^{a}$ Sites of intra-hepatic metastasis (I.M.) coupled with vascular invasion in liver, including microscopic vascular invasion in small vessels and macroscopic tumor invasion in bile duct, hepatic vein and portal vein can be observed

${ }^{b}$ Extra-hepatic metastasis (E.M.) including regional lymph node metastasis and distant organ metastasis can be observed

${ }^{\mathrm{c}}$ Total Metastasis is equal to the summation of I.M. and E.M.

${ }^{\mathrm{d}}$ The percentage in parenthesis represent the ratio of the number of HCCs with indicated PXN (p-Y31) status (ie. $T<N$ or $T>N$ ) vs total number of HCCs (all case) classified as either positive or negative of the indicated metastatic potential.

and HCC353Scramble, as negative controls. As demonstrated in Figure 4A, Hic-5 expression decreased in HCC329Hic-5(-), HCC372Hic-5(-), and HCC 353 Hic- $5(-)$ by $89 \%, 91 \%$ and $75 \%$, respectively, in comparison with that of each HCC expressing scramble shRNA. Consistently, motility of HCC329Hic-5(-), HCC372Hic-5(-), andHCC353Hic-5(-) greatly decreased by $52 \%, 91 \%$ and $35 \%$, respectively, in comparison with that of each HCC $3 x x$ Scramble (Figure 4B). Moreover, cell invasion assay using matri-gel coated cultured insert demonstrated that invasiveness of HCC329Hic-5(-) decreased by 50\% compared with that of HCC329Scramble (Supplementary Figure S4). Similar result was observed in migration assay using cultured insert without matri-gel coating (Supplementary Figure S4). Thus, Hic-5 was essential for motility and invasiveness of the HCC. Further, the role of Hic-5 in metastatic capability of HCCs was investigated using the most motile HCC 329 in a SCID mice model established previously [20]. The cells (as concentrated pellet) were injected into the middle lobe of liver followed by observation of the growth of primary tumor on middle lobe and metastatic loci on left or right lobe. As demonstrated in Figure 4C, HCC329 cells developed a substantial tumor in the middle lobe of liver two month after injection. Moreover, multiple loci of intra-hepatic metastasis (IM) can be observed on the right liver lobe, but with much less loci appearing on the left lobe. Surprisingly, a big white tumor was also observed on the abdomen (Fisher's exact test, SPSS $p<0.05, N=3$ ), indicative of extra-hepatic metastasis (Figure 4C). In contrast, in the mice inoculated with HCC329Hic-5(-), not only that the growth of primary tumor in the middle lobe of liver greatly decreased but also that no metastatic loci can be observed on both left and right lobe (Fisher's exact test, SPSS $p<0.05$, $N=3$ ) (Figure 4C). Moreover, HE staining of primary tumor (in the middle lobe) and metastatic lesion (in the right lobes) from mice inoculated with parental HCC329 showed characteristic hepatoma tissue in the form of trabeculae and cords with an irregular and dense tissue organization and deep stained nuclei (Figure 4D). Also, the extra-hepatic metastatic lesion on abdomen exhibited a more irregular and dense tissue organization composed of smaller tumor cells with deep stained nuclei. Surprisingly, a lot of unidentified whitecolored, hard, longitudinal tissues were intercalated within the extra-hepatic metastatic tumor (Figure 4D). In contrast, very homogenous and regular architecture characteristics of the normal liver tissue were observed in the normal region of middle liver lobe from mice inoculated with HCC329Hic-5(-) or the normal region 
HCC $\quad 329340353363365372374$

Hic-5 >

\author{
Ponceau S \\ stained blot
}

(B)

(C)

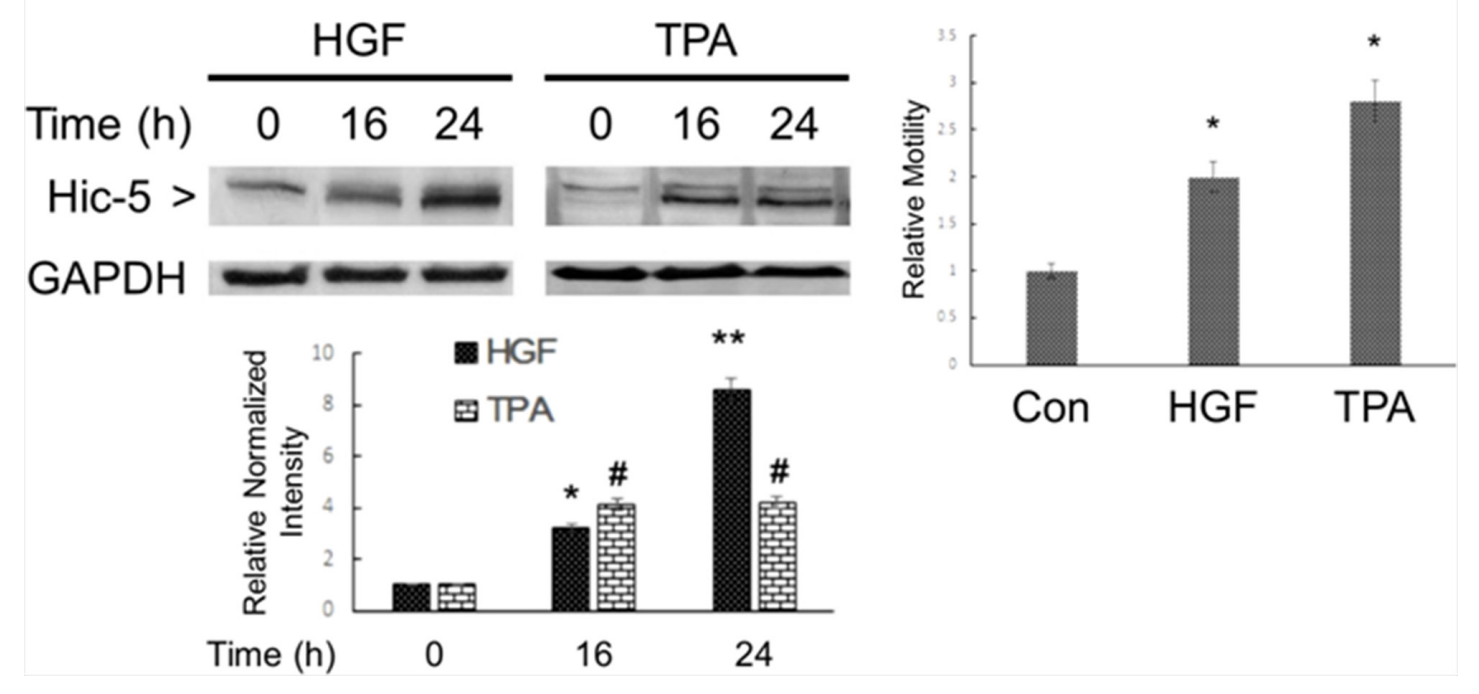

Figure 2: Constitutive and inducible expression of Hic-5 associated with motility of HCC. Various patients derived HCC was untreated (A) HCC340 was treated with HGF (left panel) and TPA (right panel) for indicated time (B) and 48 h (C) Western blot of Hic-5 (A), (B) and wound healing motility assay (C) were performed. Nonspecific bands on Ponceau S stain blot (A) and GAPDH (B) were used as loading controls. In (B) and (C) the quantitative figures demonstrate the relative intensity ratio of Hic-5/GAPDH and relative motility, respectively, taking the data of time zero (B) and untreated sample (C) as 1.0. $\left(^{* *}\right)$ and $\left(^{*}\right)\left(^{\#}\right)$ represent statistical significance $(p<$ 0.005 and $p<0.05$, respectively, $n=3$ ) for differences between the indicated samples and time zero (B) or untreated group (C).

of left lobes from mice inoculated with parental HCC329 (Figure 4D). The requirement of Hic-5 for metastatic capability of HCC 329 was validated by more than 6 reproducible animal experiments (Fisher's exact test, SPSS $p<0.05, N=8$ ), 3 of which are demonstrated in Supplementary Figure S5A. Remarkably, prominent intrahepatic and extrahepatic metastasis were observed in livers and abdomens, respectively, of mice injected with HCC329Scramble, in contrast with the dramatically reduced HCC progression in mice injected with HCC329Hic-5(-). Thus Hic-5 was required for tumor growth and intrahepatic/ extrahepatic metastasis of HCC329 in SCID mice. In addition, metastatic capability of other non-Hic-5 expressing HCCs such as HCC340 and HCC374 was very low, compared with that of HCC329 (Supplementary Figure S5B).

\section{ROS signaling is essential for Hic-5 expression in HCCs}

We further investigated the mechanistic role of Hic-5 in HCC progression focusing on the signal 


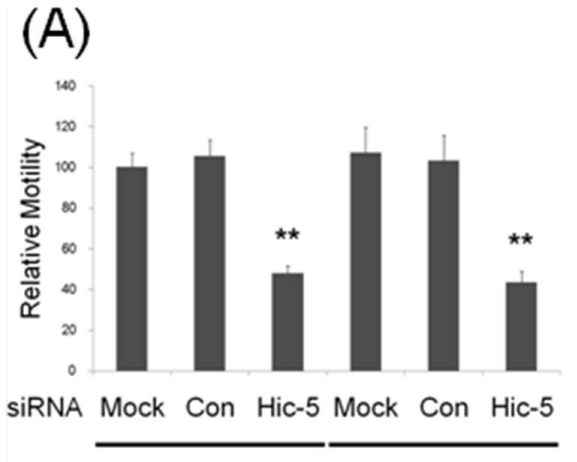

$\mathrm{HCC} 329$

(B)
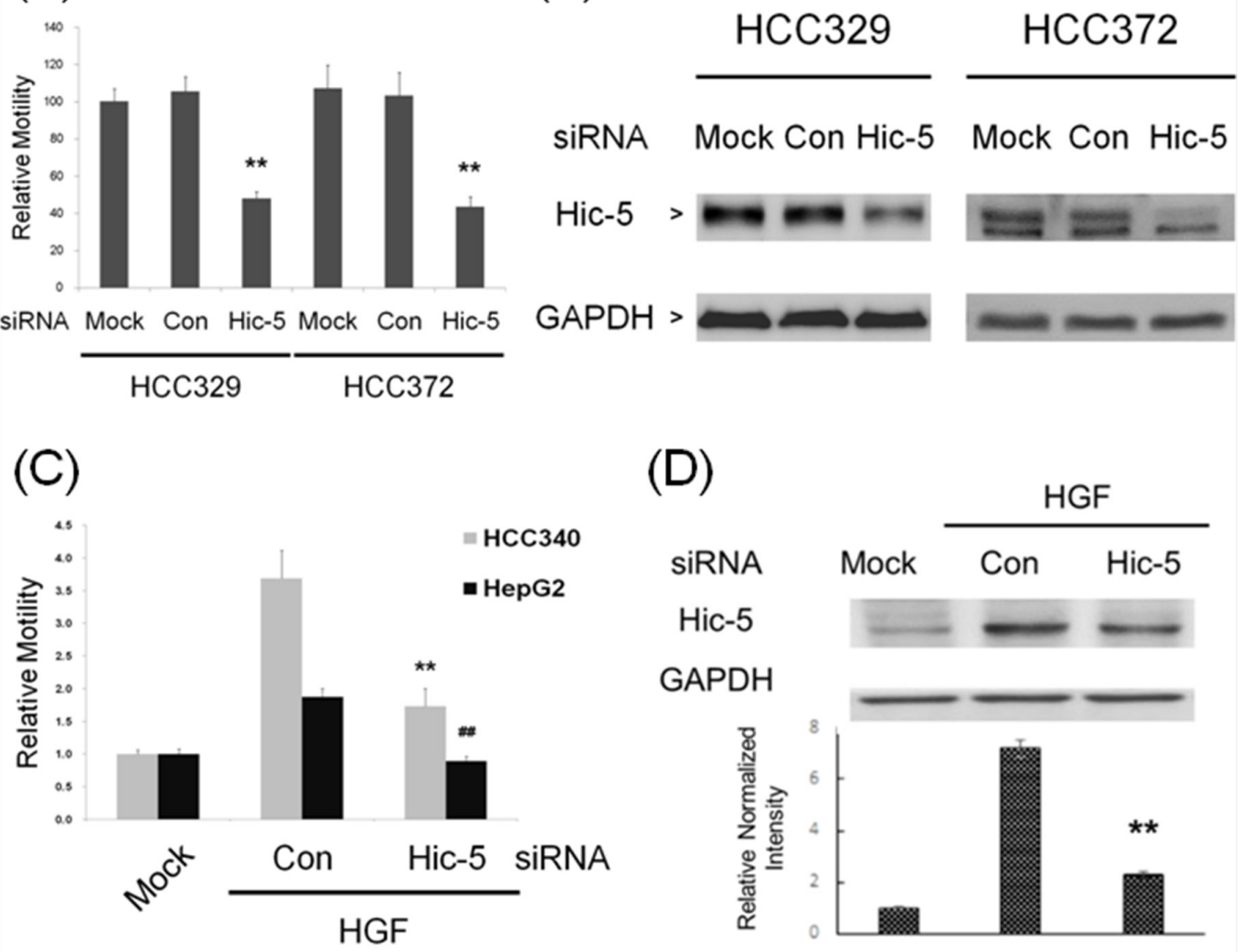

(D)

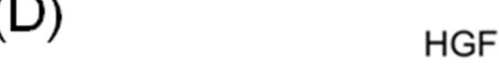

(E)

$(\mathrm{F})$
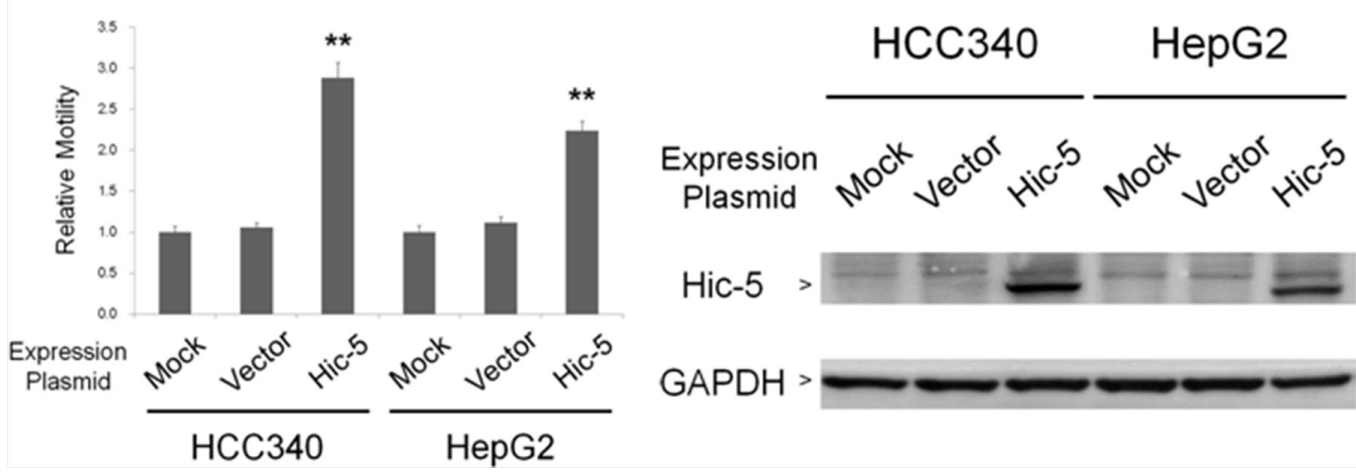

Figure 3: Hic-5 was required for constitutive and inducible HCC migration and sufficient for triggering $\mathrm{HCC}$ cell migration. HCC 329 and HCC372 were untransfected (MOCK) or transfected with Hic-5 or control (Con) siRNA (A, B) HCC340 and HepG2 were untransfected (MOCK), transfected with indicated siRNA and treated with HGF for $24 \mathrm{~h}$ (C, D) HCC340 and HepG2 were untransfected (MOCK) or transfected with Hic-5 overexpressing plasmid TGFB1I1 (E, F) Wound healing motility assay (A, C, E) and Western blot of Hic-5 (B, D, F), were performed. In (A, C, E), relative motility was calculated, taking motility of MOCK as 1.0. In (B, D, F), GAPDH was used as a loading control for the Western blots. In (D), the quantitative figure is shown below. In (A, C, D, E), $(* *)$ and $\left({ }^{(* \prime)}\right)$ represents statistical significance ( $p<0.005, n=3$ ) for difference of relative migration (A, C, E) or relative intensity (D) between the indicated groups and the control siRNA (A, C, D) or vector (E) group. (B) and (F) are representatives of two reproducible results.

pathway that Hic-5 involved. As its name indicated, Hic-5, the hydrogen peroxide inducible clone-5, was initially identified to be one of the clones in ROSinduced expression screen [15]. Moreover, ROS signaling was known to be essential for progression of a lot of tumors including HCC [23]. Therefore, we investigated whether Hic-5 expression is ROSdependent. As demonstrated in Figure 5A, treatment of $\mathrm{HCC} 329$ with the $\mathrm{H}_{2} \mathrm{O}_{2}$ degradation enzyme catalase (CAT) (500 unit/ml) decreased Hic-5 expression by 

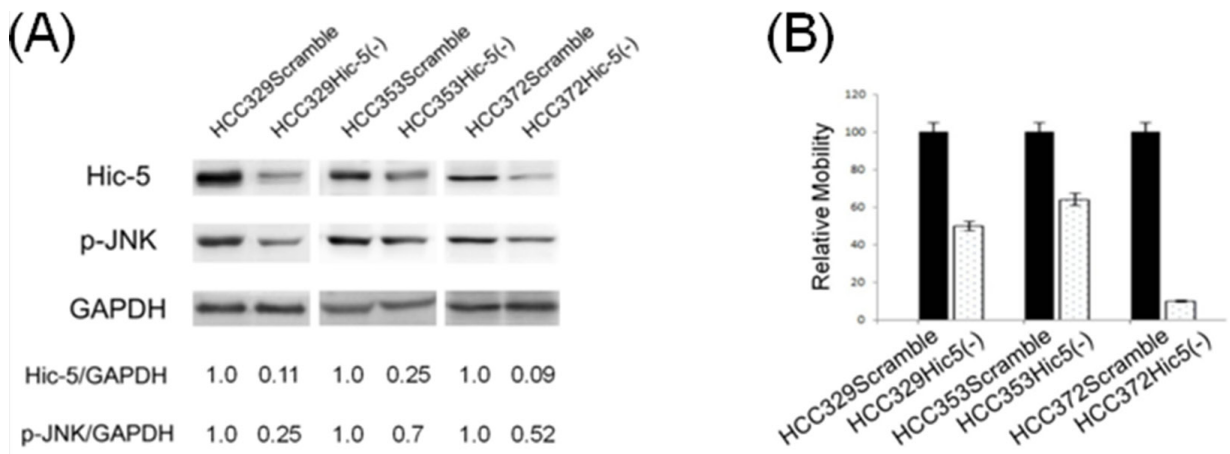

(C)

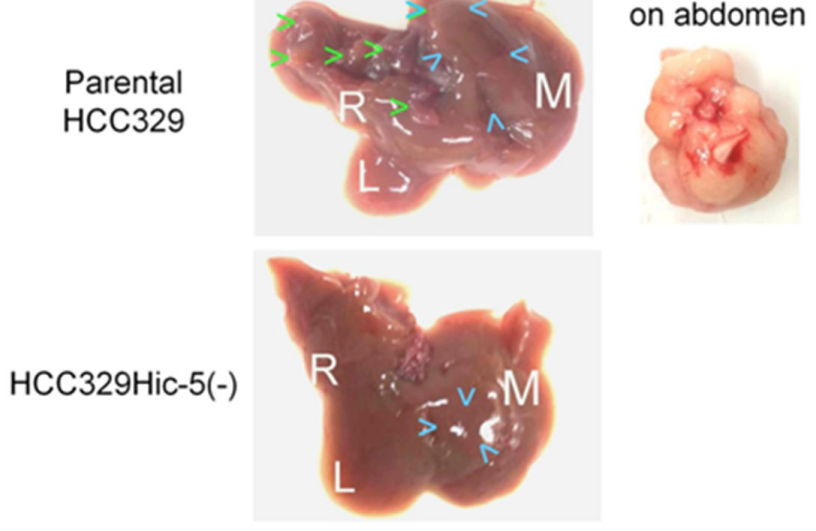

(D)

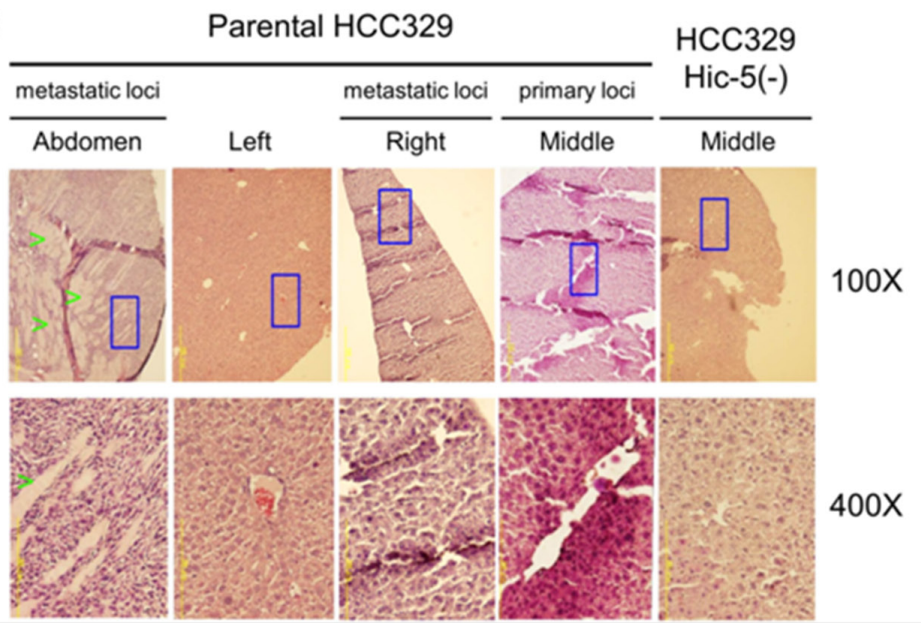

Figure 4: Depletion of Hic-5 suppressed JNK phosphorylation and progression of HCC329. Stable clone of HCCs expressing Scramble and Hic-5shRNA, denoted as HCC3xxScramble and HCC3xxHic-5(-), respectively, were obtained as indicated in Materials and Methods. Western blot of Hic-5 and phosphorylated JNK (p-JNK) (A) and cell migration (B) of indicated HCC3xxScramble and HCC3xxHic-5(-) were performed. In (A), GAPDH was used as a loading control. The numbers shown below were averaged $(N=2)$ relative intensity ratio of Hic-5/GAPDH p-JNK/GAPDH, taking the data of each HCC3xxScramble as 1.0. (B) is the quantitative figure of relative motility taking the data of each $\mathrm{HCC} 3 \mathrm{xxScramble}$ as 1.0. The data shown are average from 2 reproducible experiments. (C) Pictures of whole liver of SCID mice sacrificed after injection of each of $20 \times 10^{5}$ parental HCC329 (upper) and HCC329Hic-5(-) (lower) into middle lobe of liver for 2 months. The white letter M, L, and R represent middle, left and right liver lobes respectively. Blue and Green arrow heads indicated the location of primary and second tumors, respectively, in middle and right liver lobes. Tumor shown in the right panel was obtained from an extrahepatic metastatic lesion in abdomen. (D) H.E staining of normal tissue from HCC329Hic-5(-); primary and metastatic loci in indicated liver lobes (Middle, Right and Left) and extra metastatic loci on abdomen from parental HCC329. Blue rectangle indicates the area magnified from 100X to 400X. Green arrow head indicated the unidentified white-colored, longitudinal tissues in the extrahepatic metastatic lesion in abdomen. 
$15 \%, 35 \%$ and $62 \%$, at 4,8 , and $24 \mathrm{~h}$, respectively. Similarly, the antioxidant dithiotheritol (DTT) $(0.5 \mathrm{mM})$ decreased Hic-5 expression by $30 \%, 60 \%$ and $75 \%$, at 4, 8, and $24 \mathrm{~h}$, respectively. Consistently, CAT and DTT suppressed cell migration of HCC 329 by $40 \%$ and $60 \%$, respectively (Figure 5B). Also, treatment of HCC 353 and HCC372 with CAT and DTT for $24 \mathrm{~h}$ significantly decreased Hic-5 expression by $42-70 \%$ (Supplementary Figure S6A), consistent with the suppression of cell migration by 40-80\% (Supplementary Figure S6B). Moreover, CAT and DTT blocked HGF-induced Hic-5 expression in HCC340 by $70 \%$ (Figure 5C), consistent with the suppression of HGF-induced HCC340 cell migration by CAT and DTT by $80-100 \%$ (Figure 5D). In addition, CAT and DTT also blocked HGF-induced cell migration of HCC 363 and HepG 2 by $30-50 \%$ and $50-80 \%$, respectively (Figure 5D). ROS assay using DCF-DA labeling verified that treatment of CAT and DTT for 9-12 h decreased ROS generation by $45 \%$ and $80 \%$, respectively in HCC329 (Supplementary Figure S7). Taken together, ROS was required for constitutive and HGF-induced Hic-5 expression and cell migration of HCCs. On the other hand, $\mathrm{H}_{2} \mathrm{O}_{2}$ and the superoxide $\left(\mathrm{O}_{2}^{-2}\right)$ generator tert-butyl hydroperoxide (TBHP) induced Hic-5 expression in HCC340 by 2.5 and 4-fold, respectively, (Figure 5E), consistent with the 2.0-2.2-fold increase of cell migration (Figure 5F). These results demonstrated that ROS was also sufficient for triggering Hic-5 expression and HCC cell migration.

\section{JNK is the downstream effector of ROS required for Hic-5 expression}

In our previous studies, ROS-dependent activation of MAPK including Jun N-terminal kinase (JNK) and extracellular signal-regulated kinases (ERK), well known to mediate tumor progression [24, 25], were required for progression of HepG2 induced by TPA and $\mathrm{HGF}[8,9,19,21,22]$. Moreover, JNK activation was required for constitutive migration of HCC329 [20]. Thus we investigated the relationship of ERK and JNK with ROS-Hic-5 cascade. As demonstrated in Figure 6A, constitutive JNK phosphorylation was suppressed by DTT in HCC329 at 8 h by $74 \%$ while CAT didn't exhibit suppressive effect as DTT at this time point. However, in a more extended time course study (Supplementary Figure S8), CAT significantly suppressed JNK phosphorylation at $24 \mathrm{~h}$ (but not at 8 h) by $55 \%$ in HCC 329 . This suggests that CAT had to take longer time than DTT for suppressing JNK phosphorylation in HCC329, probably due to the slower uptake of CAT. Moreover, CAT suppressed JNK phosphorylation at $8 \mathrm{~h}$ as DTT by $60-63 \%$ in HCC 353, and the extent of inhibition exerted by both ROS scavengers was also higher (by 78-82\%) at $24 \mathrm{~h}$
(Supplementary Figure S8). In contrast, constitutive ERK phosphorylation was only marginally observed in HCC329 and not influenced by both DTT and CAT (data not shown). The suppressive effect of both ROS scavengers on MAPK activation was further examined in HGF-treated HCCs. As demonstrated in Figure 6B, JNK phosphorylation gradually increased during HGF treatment from 4 to $24 \mathrm{~h}$, with the maximal induction by 6.0 fold at $24 \mathrm{~h}$ in HCC 340. Remarkably, the HGFinduced JNK phosphorylation at $24 \mathrm{~h}$ can be blocked by CAT and DTT by $25 \%$ and $90 \%$, respectively (Figure 6B) in HCC340. Similarly, HGF-induced JNK phosphorylation at $24 \mathrm{~h}$ can be blocked by CAT and DTT by $40 \%$ and $95 \%$, respectively in HepG2 (Supplementary Figure S9). On the other hand, HGF induced transient ERK phosphorylation during 4-8 h which decreased after 16-24 h, and was not influenced by CAT and DTT at $24 \mathrm{~h}$ (not shown). Notably, the extent of HGF-induced JNK phosphorylation during 16-24 h was the most prominent within the time course, coinciding with that of HGF-induced Hic-5 expression in HCC340 (Figure 2B). Since JNK was a well known downstream signal kinase responsible for transcriptional regulation, we investigated whether $\mathrm{JNK}$ activation is also required for Hic-5 expression. As was expected, the JNK inhibitor SP600125 (SP) suppress Hic-5 expression in HCC329 (Figure 6C), HCC372 and HCC353 (Supplementary Figure S6A, lane 4 in both panels) at $24 \mathrm{~h}$ by $62 \%, 85 \%$ and $79 \%$, respectively. Moreover, HGF-induced Hic-5 expression in HCC340 (Figure 6D) and HepG2 (data not shown) can be totally suppressed by SP as efficiently as the c-Met inhibitor JNJ38877605. In contrast, it was slightly suppressed by PD98059, (the inhibitor of MEK, upstream kinase of ERK) and not influenced by worthmannin (the inhibitor of PI3-AKT) (Figure 6D). In addition, SP effectively prevented HGFinduced cell migration of HCC340, HCC363 and HepG2 (Figure 5D). Taken together, ROS-JNK cascade was required for both constitutive and HGF-induced Hic-5 expression.

\section{Hic-5 expression was essential for ROS generation and JNK phosphorylation}

One interesting study demonstrated that Hic-5 may directly associate with TRAF4/p47phox complex required for NADPH oxidase activation, and suggested a role of Hic-5 in triggering ROS generation in focal adhesion for cell migration [26]. Thus, it is tempting to observe whether Hic-5 was also essential for ROS generation in HCC. As demonstrated in Figure 7A, HCC329Hic-5(-), HCC372Hic-5(-) and HCC353Hic-5(-) exhibited decreased ROS generation by $30 \%, 70 \%$ and $35 \%$, respectively, compared with each HCCScramble. On the other hand, transfection HepG2, HCC340 and HCC363 with Hic-5 
(A)

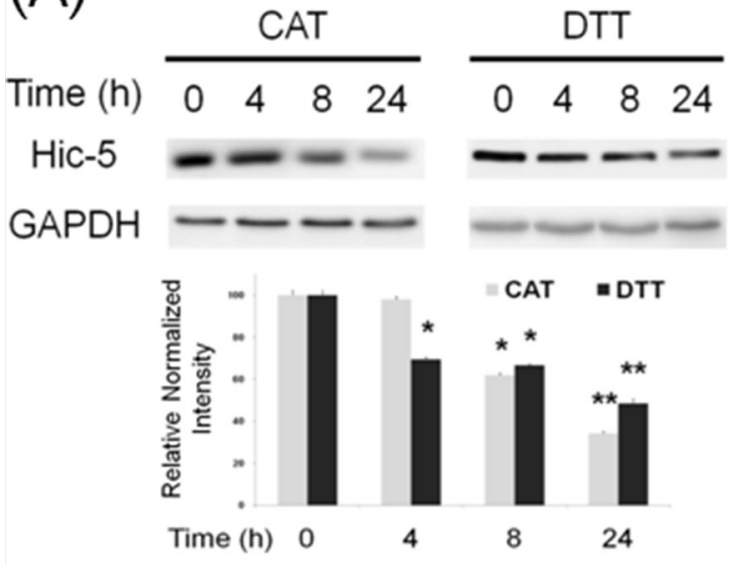

(B)

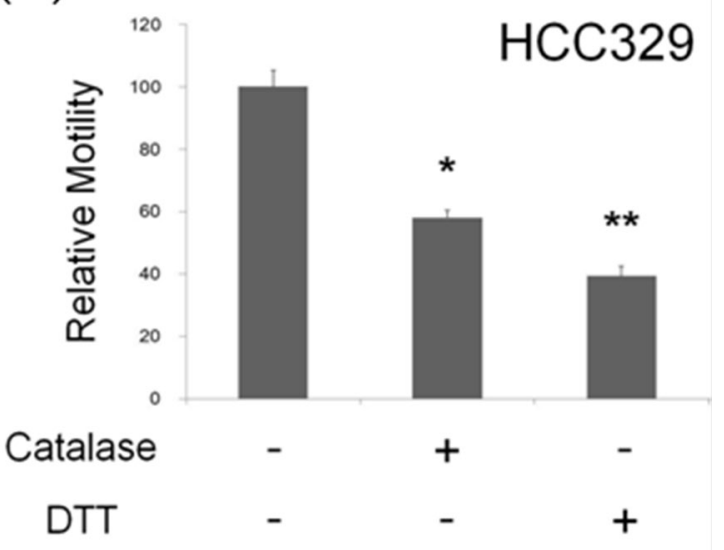

(C)

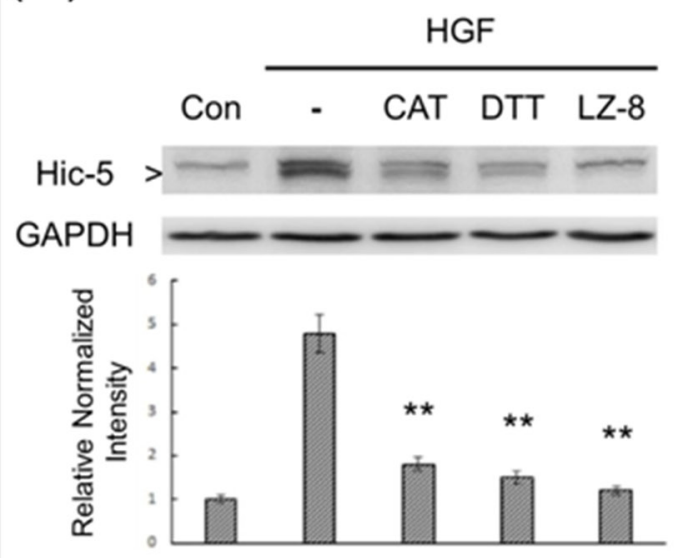

(D)

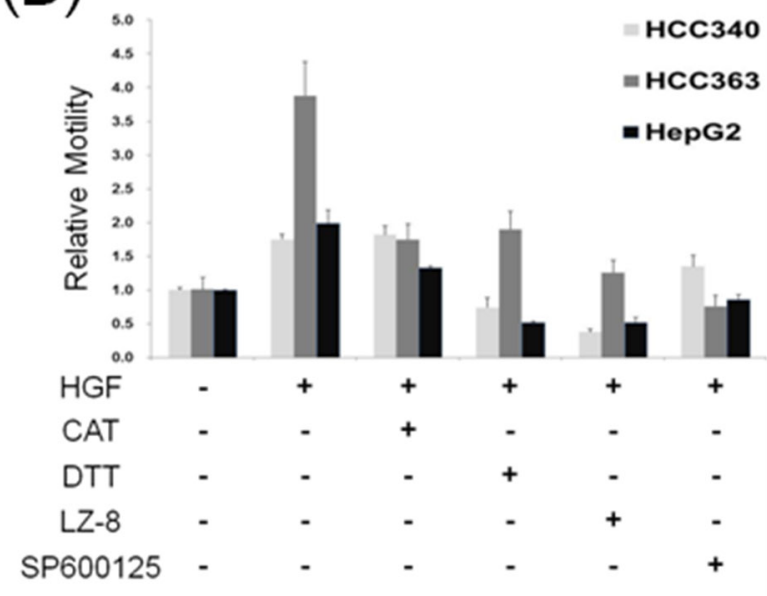

(E)

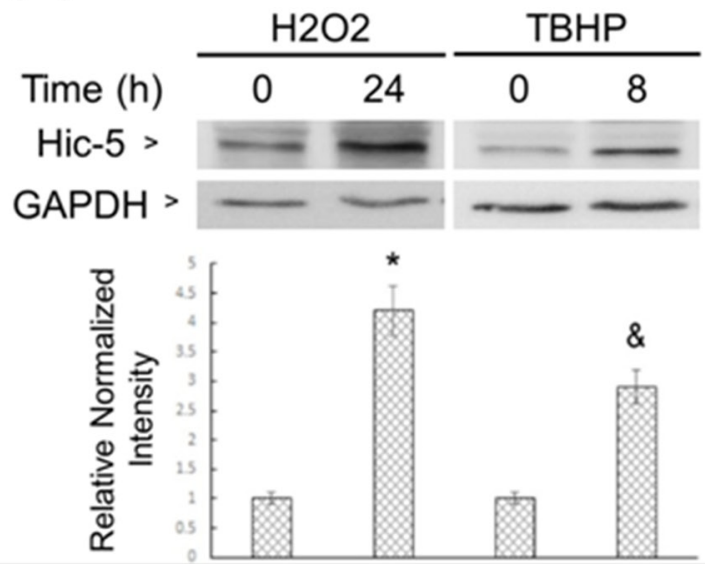

$(\mathrm{F})$

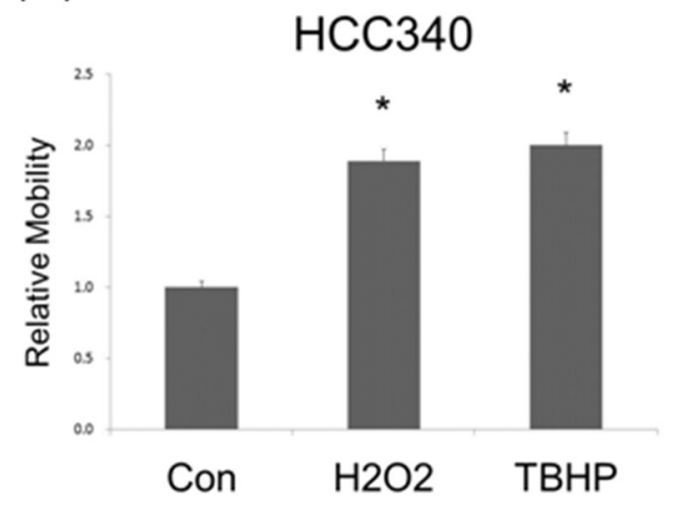

Figure 5: ROS is essential for constitutive and HGF-induced Hic-5 expression and HCC migration. HCC329 was treated with 500 unit/ml catalase (CAT) or $0.5 \mathrm{mM}$ dithiotheritol (DTT) for indicated times (A) or $48 \mathrm{~h}$ (B) HCC340 was untreated (Con), treated with HGF alone, or HGF coupled with the indicated inhibitors for $24 \mathrm{~h}$ (C) HCC340, HCC363 and HepG2 were untreated (-), treated with HGF alone, or HGF coupled with the indicated ROS scavengers, JNK inhibitor (SP600125, $20 \mu \mathrm{M})$ or LZ8 $(2.5 \mu \mathrm{g} / \mathrm{ml})$ for $48 \mathrm{~h}$ (D) HCC340 were treated with the indicated ROS generator for indicated times (E, F) Western blot of Hic-5 (A, C, E) and motility assay (B, D, F) were performed. GAPDH was used as loading control for the Western blots. The quantitative figures were demonstrated below each blot. In (B, D, F), relative motility was calculated, taking motility of the untreated sample as $100(\mathrm{~B})$ or $1.0(\mathrm{D}, \mathrm{F}) .\left({ }^{* *}\right)$ and $\left({ }^{*}\right)\left({ }^{(}\right)$ represent statistical significance ( $p<0.005$ and $p<0.05$, respectively, $n=4$ ) for differences between the indicated samples and time zero (A, E), untreated (B, F) or HGF-only (C) groups. The data shown in (D) are average from 2 reproducible experiments. 
(B)

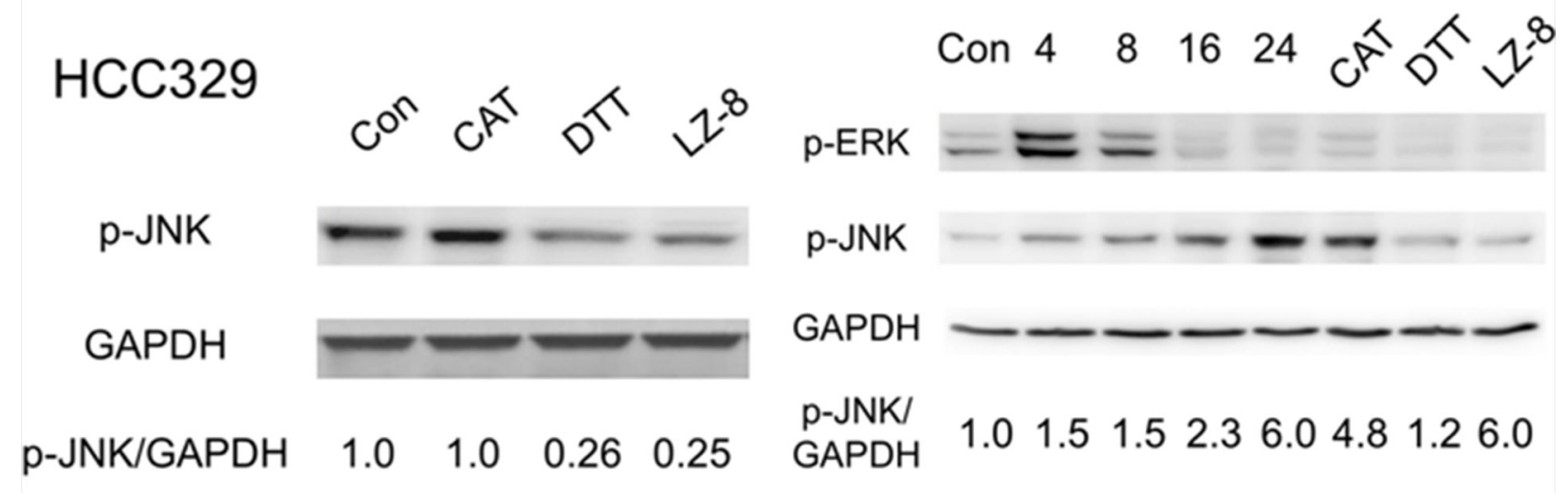

(C)

(D)
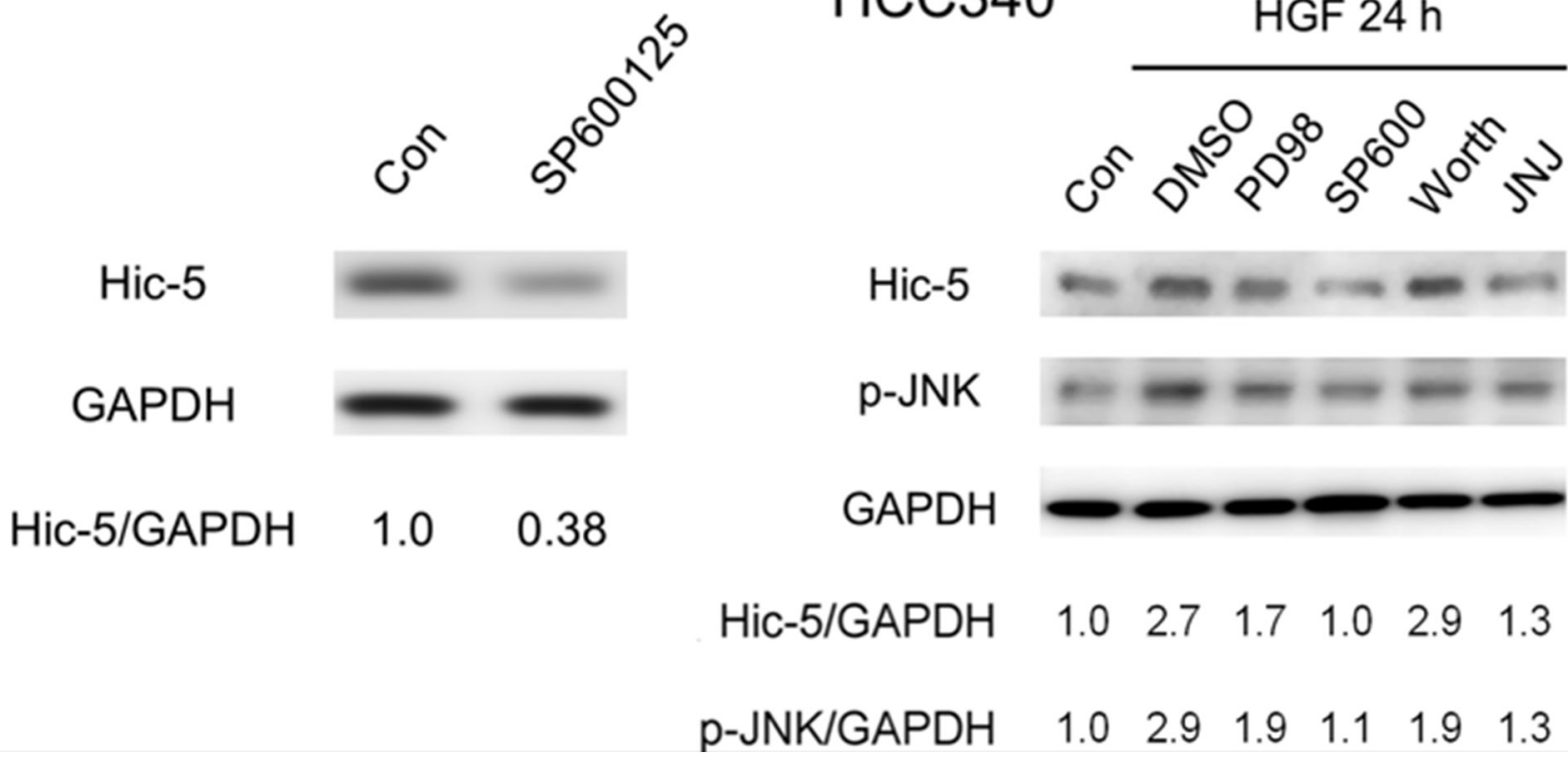

Figure 6: ROS-dependent JNK phosphorylation is required for Hic-5 expression in various HCCs. HCC329 was treated with indicated ROS scavengers or LZ-8 $(2.5 \mu \mathrm{g} / \mathrm{ml})$ (A) or JNK inhibitor SP600125 (SP) (20 $\mu \mathrm{M})$ for $24 \mathrm{~h}$ (C); HCC340 were untreated (Con), treated with HGF, or HGF coupled with indicated ROS scavenger or LZ-8 (B) or various inhibitors (D) for indicated time; Western blot of p-JNK (A), (B), (D), p-ERK (B) and Hic-5 (C, D) were performed using GAPDH as an internal control. The numbers shown below were averaged $(N=2)$ relative intensity ratio of p-JNK/GAPDH (A), (B), (D) and Hic-5/GAPDH (C), (D), taking the data of untreated (Con) as 1.0. In (D), PD98:PD98059, SP600: SP600125, Worth: Worthmannin, JNJ: JNJ38877605, a c-Met inhibitor.

expressing plasmid greatly increased ROS generation to 1.8, 4.9 and 2.2-fold (Figure 7B). In addition, blockade of Hic-5 expression by siRNA prevented HGF-induced ROS generation at $6 \mathrm{~h}$ in HepG2 (Figure 7C). Together, these indicated that Hic-5 also acted upstream of ROS. Since JNK is down stream of ROS, we further investigated whether Hic-5 was essential for JNK activation. As demonstrated in Figure 4A, phosphorylated JNK (p-JNK) greatly decreased in HCC329Hic-5(-), HCC372Hic-5(-) and $\mathrm{HCC} 353 \mathrm{Hic}-5(-)$ by $75 \%, 48 \%$ and $30 \%$ respectively, compared with that in each HCCScramble. Moreover, blockade of Hic-5 expression by Hic-5 siRNA suppressed 
HGF-induced JNK phosphorylation at $24 \mathrm{~h}$ in HCC340 and $\mathrm{HepG} 2$ by $66 \%$ and $90 \%$, respectively (Figure 7D). On the other hand, overexpression of Hic-5 significantly increased JNK phosphorylation to 2.1-fold in HCC340 (Supplementary Figure S10). Thus Hic-5 was also required for constitutive and HGF-induced JNK activation, and sufficient for triggering JNK activation in HCCs.

Collectively, ROS-JNK signaling can be both upstream and downstream of Hic-5, suggesting that Hic-5 may be responsible for establishing a positive feedback signal circuit for cell migration of HCC as elucidated in "Discussion".

\section{LZ-8 suppressed Hic-5, p-JNK and ROS generation}

We further investigated whether the ROS-Hic-5-JNK pathway can be a potential therapeutic target for preventing HCC progression. Our recent report demonstrated that LZ-8 (also known as Lingzhi-8 or Reishi), a medicinal peptide purified from the Chinese herbal drug Ganoderma lucidium, prevented $\mathrm{HCC}$ progression of $\mathrm{HCC} 329$ in vitro and in vivo [20]. Interestingly, LZ-8 (at 0.5, 2.5 and 5.0 $\mu \mathrm{g} / \mathrm{ml}$ ) greatly suppressed constitutive Hic-5 expression and JNK phosphorylation in HCC 329 by $85-60 \%$ in a dose dependent manner (Figure 8A). LZ-8 also decreased ERK phosphorylation in $\mathrm{HCC} 329$ by $40 \%$ at all concentrations. Consistently, treatment of $2.5 \mu \mathrm{M} \mathrm{LZ}-8$ for $0.5,1.5,6$ and $12 \mathrm{~h}$ suppressed constitutive ROS generation in HCC 329 by $40-55 \%$ (Figure $8 B$ ). On the other hand, LZ- 8 totally decreased the HGF-induced Hic-5 expression (Figure $5 \mathrm{C}$ ), JNK phosphorylation (Figure 6B) and cell migration (Figure 5D) of HCC340. Interestingly, increased Hic-5 expression coupled with JNK phosphorylation in HCC340 transiently transfected with Hic-5 expressing vector can be totally suppressed by LZ-8 (Supplementary Figure S10). In addition, LZ-8 can also prevent the increased cell migration of HCC 340 transiently transfected with Hic-5 expressing vector for $48 \mathrm{~h}$ (data not shown). Taken together, ROS-Hic5-JNK signaling can be blocked by LZ-8 in HCC.

\section{DISCUSSION}

\section{Hic-5 is a potential marker of HCC progression}

Previous studies demonstrated that Hic-5 overexpressed in a varieties of invasive/metastatic cancers, including breast, lung, and prostate tumors and was emerging as a potential prognostic marker [16]. In the present study, we further demonstrated the direct correlation of Hic-5 with HCC metastasis in $145 \mathrm{HCCs}$ (Table 1). The association of Hic-5 with HCC metastasis and the feasibility of Hic-5 as a HCC prognosis marker are worthy of further validation by screening more $\mathrm{HCC}$ samples.

\section{Hic-5 plays a critical role in HCC progression}

The involvement of Hic-5 in tumor progression has been mentioned previously. Hic-5 expression can be induced by TGF $\beta$ leading to epithelial mesenchymal transition (EMT), cell migration, and invasion [27]. Furthermore, ectopic expression of Hic-5 is sufficient to promote normal mammary cells to undergo EMT [27]. In addition, the melanoma cell depleted of Hic-5 exhibit decreased cell motility and metastatic activity in vivo [28]. One recent report demonstrated the upregulation of Hic5 in HCCs overexpressing proline-rich tyrosine kinase 2 (Pyk2) [29], which is known to be involved in HCC metastasis [30, 31]. Also TGF- $\beta$, which is responsible for triggering HCC progression [32, 33], can induce Hic-5 expression for malignant transformation [27]. In the present study, we further demonstrated that Hic-5 is essential for cell migration (Figure 3) and metastasis (Figure 4) of HCC.

\section{Hic-5 mediates positive feedback ROS-JNK signaling}

The diverse roles of Hic-5 in regulating signal transduction in various systems has been intensively studied [for reviews, 12, 16]. Previously, Hic-5 was found to promote TGF $\beta$-induced signaling by binding to and inactivating the inhibitory Smads, Smad3 [34] and Smad7 [35] leading to enhanced TGF- $\beta / \mathrm{Smad} 2 /$ MAPK signaling required for EMT. Hic-5 also served as a scaffold protein that specifically activates the MAPK cascade [36]. Also, ROS signaling is capable of triggering Hic-5 gene expression [15] and nuclear translocation [37]. It was well established that ROS was essential for triggering tumor progression via a lot of signal cascades including MAPK [for review, 38]. For example, ROS may activate JNK to trigger EMT [39] known to be a critical step for tumor metastasis. One recent study demonstrated Nox2-dependent ROS-JNK signaling is essential for HGF-induced mobilization of endothelial progenitor cells (EPCs), involved in proangiogenesis and tumor progression [40]. In the present study, we found Hic-5 expression is closely related with ROS-JNK signaling during HCC progression. On the one hand, ROS generation and JNK phosphorylation were required for constitutive and HGF-induced Hic-5 expression (Figure 5, 6). On the other hand, not only that Hic-5 expression is required for constitutive and HGF-induced ROS generation (Figure 7A, 7C) but also that ectopic Hic-5 expression was sufficient for triggering ROS generation (Figure 7B) and JNK phosphorylation (Supplementary Figure S10). Taken together, Hic-5 can be both upstream and down of ROSJNK, suggesting it plays the central role in establishing a positive feedback and sustained ROS and JNK signaling (see Scheme in Figure 9). The ROS and JNK signaling are known to be closely associated with sustained signal 
(A)

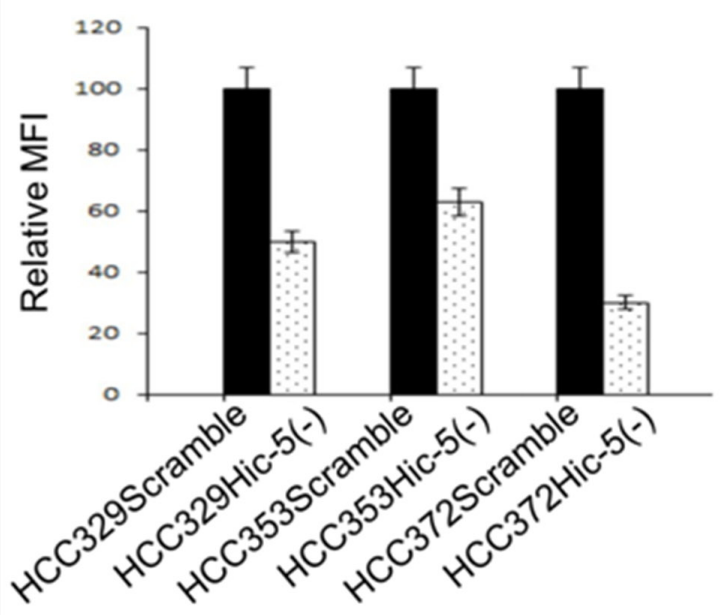

(C)

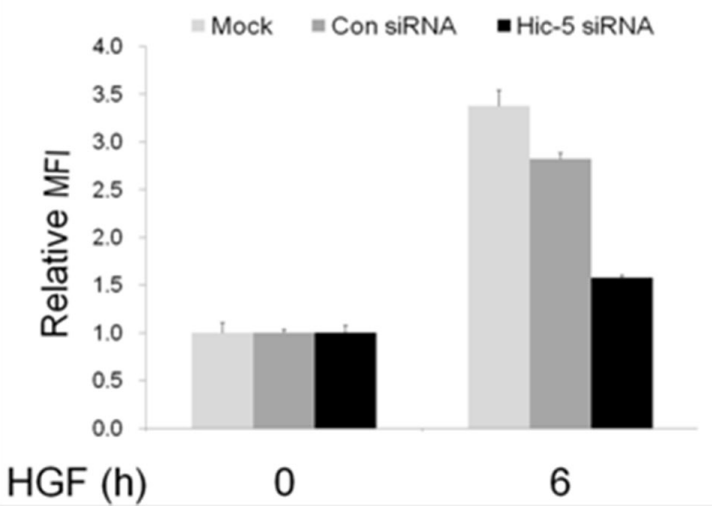

(B)

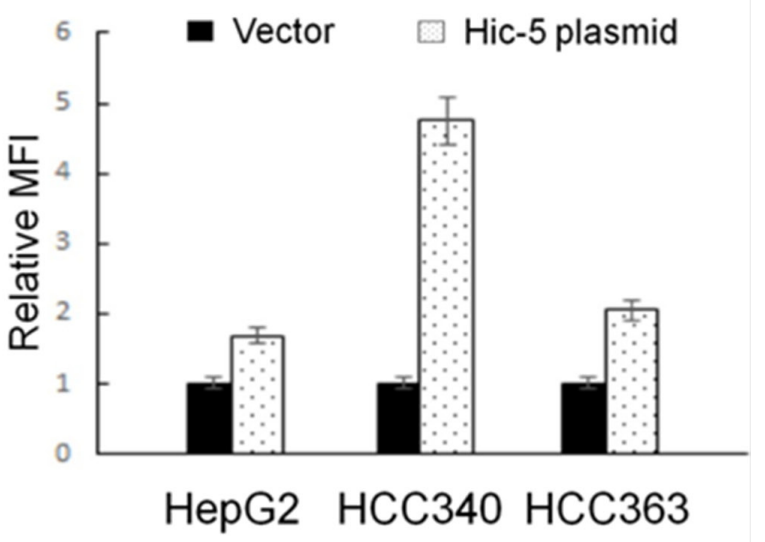

(D)

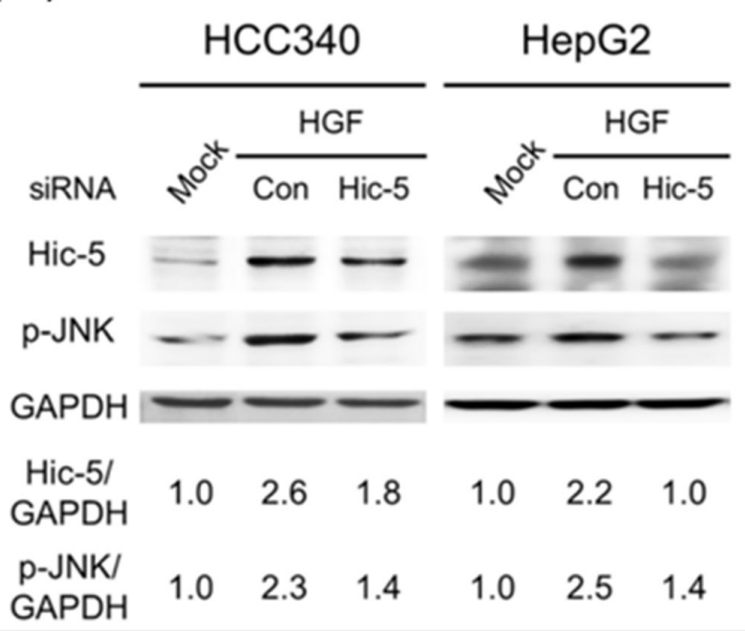

Figure 7: Hic-5 is required for constitutive and HGF-induced ROS generation and JNK phosphorylation, and sufficient for ROS generation and JNK phosphorylation. HCCs stably expressing Scramble or Hic-5 shRNA as indicated were untreated (A) HCC340 and HepG2 were transiently transfected with Hic-5 overexpressing plasmid for 48 h (B) HepG2 were untransfected and untreated (MOCK), transiently transfected with control (Con) or Hic-5siRNA and treated with HGF for indicated time (C) HCC340 and HepG2 were untransfected (MOCK), transfected with Hic-5siRNA and treated with HGF for indicated time (D) ROS generation assay (A), B), and (C) and Western blot of Hic-5 and phosphorylated JNK (p-JNK) (D) were performed using GAPDH as an internal control. In (A), (B) and (C), MFI: mean fluorescence intensity representing the G mean of DCF fluorescence detected in flow cytometry. Relative MFI was calculated, taking HCCScramble (A), HCC transfected with vector (B) and control siRNA group (C) as 100 or 1.0 as indicated. The data shown are average from 2 reproducible experiments. In (D), the numbers shown below were averaged $(N=2)$ relative intensity ratio of $\mathrm{p}-\mathrm{JNK} / \mathrm{GAPDH}$ and Hic-5/GAPDH, taking the data of MOCK as 1.0.

transduction for a lot of patho-physiological processes. For example, ROS are responsible for signal cross talks that triggers sustained MAPK activation and cell migration [41]. Also, ROS is critical for positive feedback loop triggering the sustained activation of Akt, which lead to mesangial cell hypertrophy and diabetic nephropathy [42].
On the other hand, sustained JNK signaling was required for Cylindromatosis (CYLD) (a deubiquitination enzyme) induced c-MYC expression and histone $\mathrm{H} 3$ methylations for HCC progression [43]. The detailed mechanism for Hic-5 to mediate sustained ROS-JNK signaling and HCC progression is worthy of further investigation. 
(A)
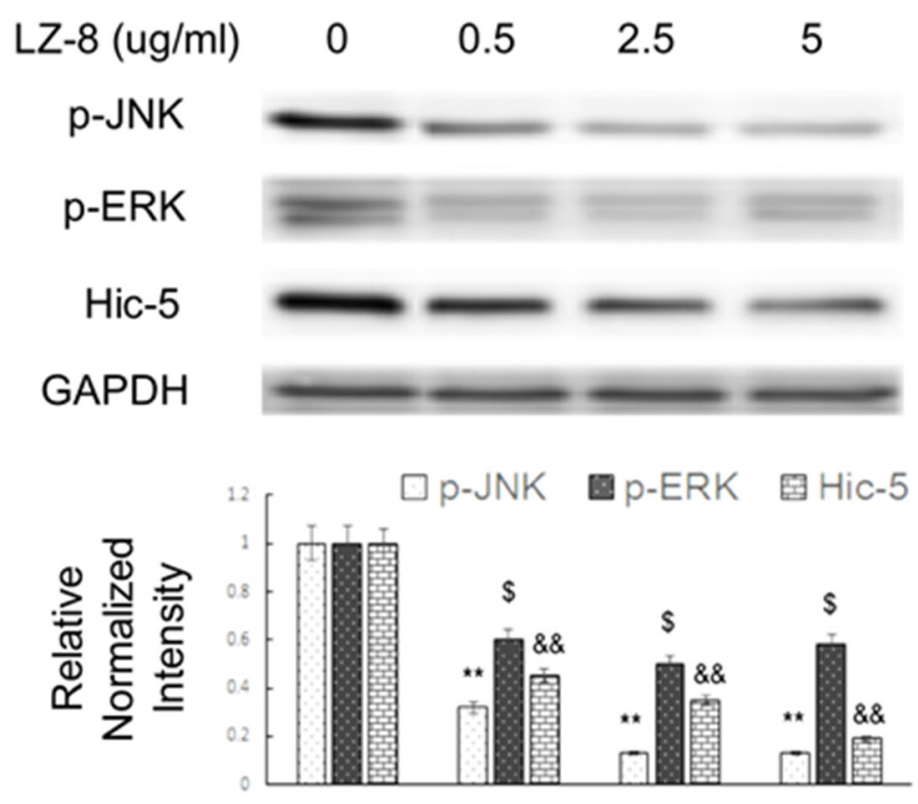

(B)

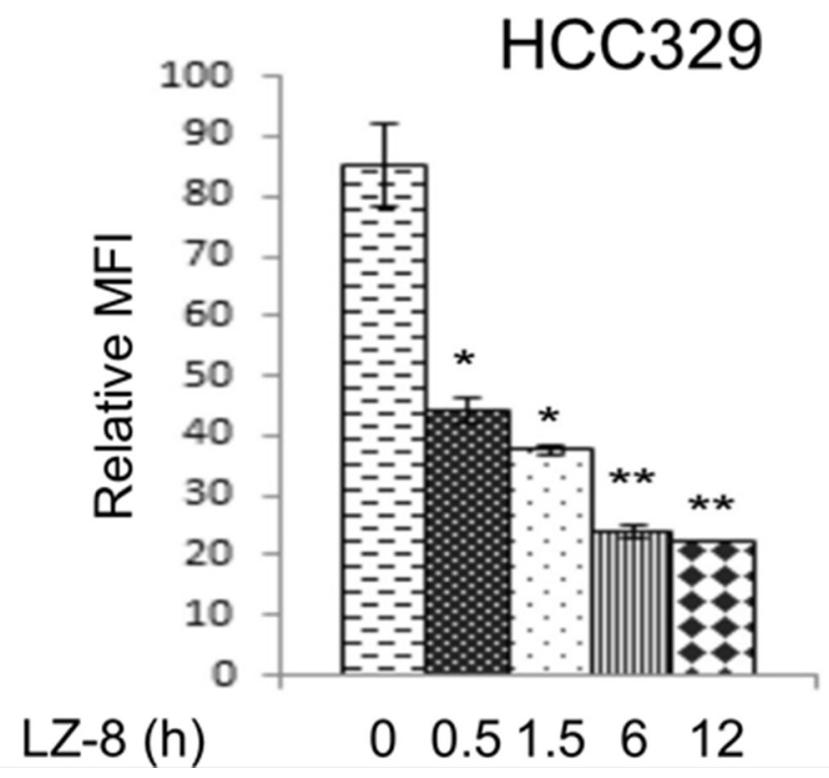

Figure 8: LZ-8 suppressed constitutive expression of Hic-5, JNK activation and ROS generation in HCC329. HCC329 were treated with LZ-8 at indicated concentration for $24 \mathrm{~h}$ (A) HCC329 were treated with $2.5 \mu \mathrm{g} / \mathrm{ml} \mathrm{LZ}-8$ at indicated time (B) Western blot of indicated molecule (A) and ROS assay (B) were performed. In (A), GAPDH were used as an internal control. Quantitative figures were demonstrated below. In (B), MFI: mean fluorescence intensity representing the G mean of DCF fluorescence detected in flow cytometry. $\left({ }^{* *}\right)\left({ }^{\& \&}\right)$ and $\left({ }^{*}\right)\left({ }^{\S}\right)$ represent statistical significance $(p<0.005$ and $p<0.05$, respectively, $n=4)$ for differences of intensity between the indicated groups and zero concentration (A) or time zero (B) group.

\section{Hic-5 is a more ideal target for prevention of tumor progression}

According to previous studies, both Hic-5 and paxillin are promising therapeutic targets for anti-HCC progression.
However, target therapy aiming at paxillin seems unfeasible due to its ubiquitous tissue expression playing essential role for diverse biological functions [16]. In contrast, Hic-5 is enriched only in certain tissue such as smooth muscle and large intestine [16], thus may be a more suitable therapeutic target. 


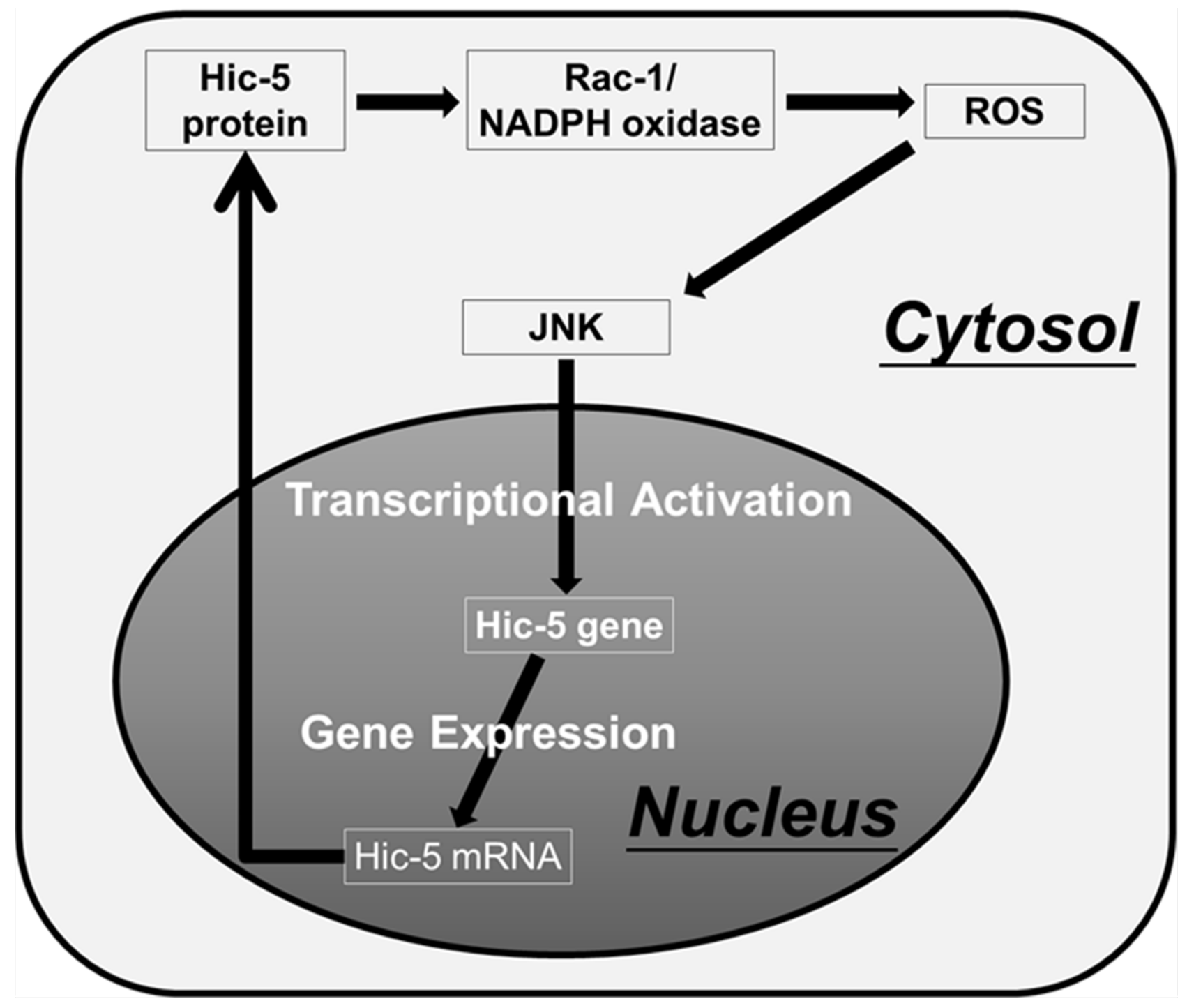

Figure 9: Proposed model for Hic-5-mediated sustained ROS-JNK signaling. Based on inhibitor studies, ROS generation and JNK phosphorylation were required for constitutive and HGF-induced Hic-5 expression (Figure 5-6). Also, ectopic Hic-5 expression was sufficient for triggering ROS generation (Figure 7B) and JNK phosphorylation (Supplementary Figure S10). Taken together, Hic-5 can be both upstream and down of ROS-JNK. It can be proposed that the ROS triggered-JNK activation can induce transcriptional upregulation of Hic-5. The Hic-5 protein induced in turn activates Rac-1/NADPH oxidase dependent ROS generation [26] and JNK phosphorylation thus sustaining the signal transduction and Hic-5 expression. Accordingly, Hic-5 may play a central role in mediating the positive feedback ROS-JNK signaling circuit.

\section{LZ-8 suppressed HCC progression via blocking ROS-Hic-5-JNK signaling}

In previous studies, LZ-8 was found to be both an immunomodulatory [44] and anti-tumor agent $[45,46]$. On the signal level, LZ-8 may suppress the protein kinase C-dependent pathway [47] known to be involved in HCC progression triggered by HGF-c-Met [8]. Recently, we also found LZ-8 suppress HCC progression via blocking c-Met or c-Met-independent MAPK signaling [20]. In this study, we further found LZ-8 suppressed constitutive and HGF-induced expression of Hic-5 coupled with decrease of reactive oxygen species (ROS) generation and phosphorylation of JNK and ERK in HCCs. The detailed mechanisms for how LZ-8 suppressed ROS generation that block Hic5-MAPK signaling is worthy of further investigation.

In conclusion, we found the paxillin paralog Hic5 plays an essential role in signal transduction for HCC progression and may serve as a promising prognosis marker and therapeutic target for management of HCC.

\section{MATERIALS AND METHODS}

\section{Cell lines, hepatocellular carcinoma tissue collection, plasmid and chemicals}

Human hepatoma cell HepG2 was purchased from the Bioresource Collection and Research Center 
(Hsinchu, Taiwan). HCC tissues were collected during HCC surgery at Tzu Chi Hospital with patient's consents, approved by the Research Ethics Committee in Buddhist Tzu Chi General Hospital (IRB 101$62)$. The tissues were snap frozen at $-80^{\circ} \mathrm{C}$ before being harvested for Western blotting or sectioning for immunohistochemical analysis. HGF was obtained from Peprotech (Rocky Hill, NJ, USA). TPA, dithiotheritol, catalase and SP600125, PD98059 and JNJ38877605 were from Sigma (Milwaukee, DC, USA). Antibodies for Hic-5, phosphorylated JNK (p-JNK), phosphorylated ERK (p-ERK) and GAPDH were obtained from Santa Cruz Biotechnology, Inc. (California, USA). Antibody for Hic-5 was also obtained from GeneTex (Irvine, CA, USA). Hic-5 expression plasmid (TGFB 1I1) was from OriGene Technologies, Inc. (Washington, DC, USA). LZ-8 was from Yeastern Biotech Co., Ltd. (Taipei, Taiwan).

\section{Establishing patient-derived hepatocellular carcinoma cell lines}

Clinically derived HCC cell lines were established from parts of HCC tissues obtained from surgery with patient's consents, approved by Buddhist Tzu Chi general hospital Research Ethic Committee (IRB 101-62). Briefly, HCC tissues were pretreated with collagenase and cultivated on the mitomycin C-treated NIH3T3 feeder layer for 4 to 6 passages to select the HCC cell lines. Homogenous HCC cell populations with the sustained proliferation ability (over 20 passages) were obtained. The characteristics of the HCC tumor cell lines were validated by detecting HCC tumor makers, such as Glypican 3 (GPC3) [18], after more than 40 passages.

\section{Immunohistochemistry}

Immunohistochemistry (IHC) for Hic-5 was performed according to the standard protocols established by the Research Centre for Hepatology at Tzu Chi Hospital.

\section{Wound healing migration assay}

Wound healing migration assay were performed according to our previous studies [8,9]. Quantitation of cell motility was performed by counting the cells that have migrated into the blanking area using Image $\mathrm{J}$ software.

\section{Transwell migration/invasion assay}

Cells were seeded on a 24-well transwell migration insert with (for invasion) or without (for migration) matrigel coating (Nalge Nunc International, Rochester, NY, USA) in a complete medium for $24 \mathrm{~h}$.
After appropriate treatments, cells that had migrated or invaded through the matrigel to the underside of the insert membrane were stained with $0.3 \%$ crystal violet. The cells on the topside of the insert membrane were rubbed with a cotton swab. The migrated/invaded cells on the underside were imaged using phase contrast microscopy with $200 \mathrm{X}$ magnification.

\section{Western blot}

Western blots were performed according to our previous studies $[8,9]$. The band intensities on the blots were quantified using Image $\mathrm{J}$ software.

\section{Flow cytometric analysis for ROS generation}

ROS assay was performed as described in our previous report [19]. Each determination is based on the mean fluorescence intensity (MFI) of 5,000 cells.

\section{RNA interference and establishment of cells stably depleted of Hic-5}

Hic-5 expression was transiently knocked down by transfection of the cells with $25 \mathrm{nM}$ Hic-5 siRNA (Thermo Scientific, Dharmacon, US) for $48 \mathrm{~h}$, according to the manufacture's protocol. To obtain stable clones expressing Hic-5 shRNA, lentiviral plasmids encoding shRNA for Hic-5 were packaged into 293T cell. Subsequently, the viral particle-containing mediums were used to infect HCCs followed by selection with puromycin for 2-3 weeks.

\section{Establishing hepatocellular carcinoma metastasis in SCID mice}

The metastasis of HCC was established using Nod SCID mice as previously [8,9]. All the mice were males, 8 weeks old, and had an average body weight of 35-40 g HCC cells $\left(2 \times 10^{6}\right)$ were suspended in $100 \mu \mathrm{L}$ DMEM and directly injected into the subserosa of the middle liver lobe under anesthesia. Two to four months after inoculation, the mice were sacrificed for examining the primary tumor growth on middle liver lobe and secondary tumor foci on left and right lobes. Nodules with diameters exceeding 0.1 to $0.2 \mathrm{~cm}$ on the left or right lobes were denoted as secondary tumor foci. Intrahepatic metastasis was defined if a minimum of two secondary tumor foci can be observed in the left and/or right liver lobes. Extrahepatic metastasis was defined by tumors appearing in organs other than the liver. During animal experiment, which was approved by the Institutional Animal Care and Use Committee at Tzu Chi University (No. 102080), regulations relevant to the care and use of laboratory animals were followed. 


\section{Statistical analysis}

Anova test was conducted to analyze the intensity differences between samples on the Western blot and the differences in cell motility between the indicated HCCs. Quantitative data were expressed as mean \pm coefficient variation $(\mathrm{CV})$, indicated by the error bars in each figure. The correlation of indicated molecules with HCC metastasis was analyzed by Chi-square test (SPSS 16.0 software, Chicago, IL, USA). The differences of HCC progression between mice inoculated with various HCCs were analyzed by Fisher's exact test (SPSS 16.0 software, Chicago, IL, USA).

\section{Abbreviations}

Hic-5, Hydrogen peroxide inducible clone-5; ROS, reactive oxygen species; DTT, dithiotheritol; CAT, catalase; HGF, hepatocyte growth factor; IHC, Immunohistochemistry; PXN (p-Y31), Tyr31 phosphorylated paxillin; I.M., intrahepatic metastasis; E.M., extrahepatic metastasis; JNK, Jun N-terminal kinase; ERK, extracellular signal-regulated kinases; TPA, 12-O-tetradecanoyl-phorbol-13-acetate; LZ-8, Lingzhi-8.

\section{ACKNOWLEDGMENTS AND FUNDING}

We thank Ministry of Science and Technology in Taiwan and Research Centre for Hepatology in Buddhist Tzu Chi General Hospital for financial support and Ms. Pei-Ling Ma for technical assistance.

\section{CONFLICTS OF INTEREST}

There is no Conflict of Interests to disclose.

\section{REFERENCES}

1. Tang ZY, Ye SL, Liu YK, Qin LX, Sun HC, Ye QH, Wang L, et al. A decade's studies on metastasis of hepatocellular carcinoma. J Cancer Res Clin Oncol. 2004; 130:187-196.

2. Mazzocca A, Antonaci S, Giannelli G. The TGF-beta signaling pathway as a pharmacological target in a hepatocellular carcinoma. Curr Pharm Des. 2012; 18:4148-4154.

3. Goyal L, Muzumdar MD, Zhu AX. Targeting the HGF/cMET pathway in hepatocellular carcinoma. Clin Cancer Res. 2013; 19:2310-2318.

4. Giordano S, Columbano A. Met as a therapeutic target in HCC: facts and hopes. J Hepatol. 2013; 60:442-452.

5. Brown MC, Turner CE. Paxillin: adapting to change. Physiol Rev. 2004; 84:1315-1339.

6. Deakin NO, Turner CE. Paxillin comes of age. J Cell Sci. 2008; 121:2435-2444.
7. Turner CE. Paxillin interactions. J Cell Sci. 2000; 23:4139-4140.

8. Hu CT, Cheng CC, Wu JR, Pan SM, Wu WS. PKCepsilonmediated c-Met endosomal processing directs fluctuant c-Met-JNK-paxillin signaling for tumor progression of HepG2. Cell Signal. 2015 (in press).

9. Hu CT, Cheng CC, Pan SM, Wu JR, Wu WS. PKC mediates fluctuant ERK-paxillin signaling for hepatocyte growth factor-induced migration of hepatoma cell HepG2. Cell Signal. 2013; 25:1457-1467.

10. Tang J, Wu YM, Zhao P, Yang XM, Jiang JL, Chen ZN. Overexpression of HAb18G/CD147 promotes invasion and metastasis via alpha3beta1 integrin mediated FAK-paxillin and FAK-PI3K-Ca2+ pathways. Cell Mol Life Sci. 2008; 65:2933-2942.

11. Ching YP, Leong VY, Lee MF, Xu HT, Jin DY, Ng IO. $\mathrm{P} 21$-activated protein kinase is overexpressed in hepatocellular carcinoma and enhances cancer metastasis involving c-Jun NH2-terminal kinase activation and paxillin phosphorylation. Cancer Res. 2007; 67:3601-3608.

12. Shibanuma M, Mori K, Nose K. HIC-5: A Mobile Molecular Scaffold Regulating the Anchorage Dependence of Cell Growth. Int J Cell Biol. 2012; 2012:426138.

13. Huang C, Rajfur Z, Borchers C, Schaller MD, Jacobson K. JNK phosphorylates paxillin and regulates cell migration. Nature. 2003; 424:219-223.

14. Shibanuma M, Mashimo J, Kuroki T, Nose K. Characterization of the TGF beta 1-inducible hic-5 gene that encodes a putative novel zinc finger protein and its possible involvement in cellular senescence. J Biol Chem. 1994; 269:26767-26774.

15. Nose K. Regulation of gene expression by active oxygen species. Yakugaku Zasshi. 2002; 122:773-780.

16. Deakin NO, Pignatelli J, Turner CE. Diverse roles for the paxillin family of proteins in cancer. Genes Cancer. 2012; $3: 362-370$.

17. Deakin NO, Turner CE. Distinct roles for paxillin and Hic-5 in regulating breast cancer cell morphology, invasion, and metastasis. Mol Biol Cell. 2011; 22:327-341.

18. Zhu AX, Gold PJ, El-Khoueiry AB, Abrams TA, Morikawa H, Ohishi N, Ohtomo T, et al. First-in-man phase I study of GC33, a novel recombinant humanized antibody against glypican-3, in patients with advanced hepatocellular carcinoma. Clin Cancer Res. 2013; 19:920-928.

19. $\mathrm{Hu} \mathrm{CT}, \mathrm{Wu} \mathrm{JR}$, Cheng CC, Wang S, Wang HT, Lee MC, Wang LJ, et al. Reactive oxygen species-mediated $\mathrm{PKC}$ and integrin signaling promotes tumor progression of human hepatoma HepG2. Clin Exp Metastasis. 2011; 28:851-863.

20. Wu JR, Hu CT, You RI, Ma PL, Pan SM, Lee MC, $\mathrm{Wu}$ WS. Preclinical trials for prevention of tumor progression of hepatocellular carcinoma by LZ-8 targeting c-Met 
dependent and independent pathways. PLoS One. 2015; 10:e0114495.

21. Wu WS, Tsai RK, Chang CH, Wang S, Wu JR, Chang YX. Reactive oxygen species mediated sustained activation of protein kinase $\mathrm{C}$ alpha and extracellular signal-regulated kinase for migration of human hepatoma cell Hepg2. Mol Cancer Res. 2006; 4:747-758.

22. Hu CT, Wu JR, Chang TY, Cheng CC, Wu WS. The transcriptional factor Snail simultaneously triggers cell cycle arrest and migration of human hepatoma HepG2. J Biomed Sci. 2008; 15:343-355.

23. Block K, Gorin Y. Aiding and abetting roles of NOX oxidases in cellular transformation. Nat Rev Cancer. 2012; 12:627-637.

24. Fang JY, Richardson BC. The MAPK signalling pathways and colorectal cancer. Lancet Oncol. 2005; 6:322-327.

25. Reddy KB, Nabha SM, Atanaskova N. Role of MAP kinase in tumor progression and invasion. Cancer Metastasis Rev. 2003; 22:395-403.

26. $\mathrm{Wu} \mathrm{RF}, \mathrm{Xu} \mathrm{YC}, \mathrm{Ma} \mathrm{Z}$, Nwariaku FE, Sarosi GA Jr., Terada LS. Subcellular targeting of oxidants during endothelial cell migration. The Journal of cell biology. 2005; 171:893-904.

27. Pignatelli J, Tumbarello DA, Schmidt RP, Turner CE. Hic-5 promotes invadopodia formation and invasion during TGFbeta-induced epithelial-mesenchymal transition. J Cell Biol. 2012; 197:421-437.

28. Noguchi F, Inui S, Nakajima T, Itami S. Hic-5 affects proliferation, migration and invasion of B16 murine melanoma cells. Pigment Cell Melanoma Res. 2012; 25:773-782.

29. Sun CK, Ng KT, Lim ZX, Cheng Q, Lo CM, Poon RT, Man K, et al. Proline-rich tyrosine kinase 2 (Pyk2) promotes cell motility of hepatocellular carcinoma through induction of epithelial to mesenchymal transition. PLoS One. 2011; 6:e18878.

30. Liu RF, Xu X, Huang J, Fei QL, Chen F, Li YD, Han ZG. Down-regulation of miR-517a and miR-517c promotes proliferation of hepatocellular carcinoma cells via targeting Pyk2. Cancer Lett. 2013; 329:164-173.

31. Geng W, Ng KT, Sun CK, Yau WL, Liu XB, Cheng Q, Poon RT, et al. The role of proline rich tyrosine kinase 2 (Pyk2) on cisplatin resistance in hepatocellular carcinoma. PLoS One. 2011; 6:e27362.

32. Dhanasekaran R, Nakamura I, Hu C, Chen G, Oseini AM, Seven ES, Miamen AG, et al. Activation of the transforming growth factor-beta/SMAD transcriptional pathway underlies a novel tumor-promoting role of sulfatase 1 in hepatocellular carcinoma. Hepatology. 2014; 61:1269-1283.

33. Reichl P, Dengler M, van Zijl F, Huber H, Fuhrlinger G, Reichel C, Sieghart W, et al. Axl activates autocrine transforming growth factor-beta signaling in hepatocellular carcinoma. Hepatology. 2014; 61:930-941.

34. Wang H, Song K, Sponseller TL, Danielpour D. Novel function of androgen receptor-associated protein 55/Hic-5 as a negative regulator of Smad3 signaling. J Biol Chem. 2005; 280:5154-5162.

35. Wang H, Song K, Krebs TL, Yang J, Danielpour D. Smad7 is inactivated through a direct physical interaction with the LIM protein Hic-5/ARA55. Oncogene. 2008; 27:6791-6805.

36. Lei XF, Kim-Kaneyama JR, Arita-Okubo S, Offermanns S, Itabe H, Miyazaki T, Miyazaki A. Identification of Hic-5 as a novel scaffold for the MKK4/p54 JNK pathway in the development of abdominal aortic aneurysms. J Am Heart Assoc. 2014; 3:e000747.

37. Shibanuma M, Kim-Kaneyama JR, Ishino K, Sakamoto N, Hishiki T, Yamaguchi K, Mori K, et al. Hic-5 communicates between focal adhesions and the nucleus through oxidant-sensitive nuclear export signal. Mol Biol Cell. 2003; 14:1158-1171.

38. Wu WS. The signaling mechanism of ROS in tumor progression. Cancer Metastasis Rev. 2006; 25:695-705.

39. Fukawa T, Kajiya H, Ozeki S, Ikebe T, Okabe K. Reactive oxygen species stimulates epithelial mesenchymal transition in normal human epidermal keratinocytes via TGF-beta secretion. Experimental cell research. 2012; 318:1926-1932.

40. Schroder K, Schutz S, Schloffel I, Batz S, Takac I, Weissmann N, Michaelis UR, Koyanagi M, Brandes RP. Hepatocyte growth factor induces a proangiogenic phenotype and mobilizes endothelial progenitor cells by activating Nox2. Antioxidants \& redox signaling. 2011; 15:915-923.

41. Wu WS, Wu JR, Hu CT. Signal cross talks for sustained MAPK activation and cell migration: the potential role of reactive oxygen species. Cancer metastasis reviews. 2008; 27:303-314.

42. Das F, Ghosh-Choudhury N, Dey N, Bera A, Mariappan MM, Kasinath BS, Ghosh Choudhury G. High glucose forces a positive feedback loop connecting Akt kinase and FoxO1 transcription factor to activate mTORC1 kinase for mesangial cell hypertrophy and matrix protein expression. The Journal of biological chemistry. 2014; 289:32703-32716.

43. Pannem RR, Dorn C, Ahlqvist K, Bosserhoff AK, Hellerbrand C, Massoumi R. CYLD controls c-MYC expression through the JNK-dependent signaling pathway in hepatocellular carcinoma. Carcinogenesis. 2014; 35:461-468.

44. Xue Q, Ding Y, Shang C, Jiang C, Zhao M. Functional expression of LZ-8, a fungal immunomodulatory protein 
from Ganoderma lucidium in Pichia pastoris. J Gen Appl Microbiol. 2008; 54:393-398.

45. Martinez-Montemayor MM, Acevedo RR, Otero-Franqui E, Cubano LA, Dharmawardhane SF. Ganoderma lucidum (Reishi) inhibits cancer cell growth and expression of key molecules in inflammatory breast cancer. Nutr Cancer. 2011; 63:1085-1094.

46. Liao CH, Hsiao YM, Hsu CP, Lin MY, Wang JC, Huang YL, Ko JL. Transcriptionally mediated inhibition of telomerase of fungal immunomodulatory protein from Ganoderma tsugae in A549 human lung adenocarcinoma cell line. Mol Carcinog. 2006; 45:220-229.

47. Hsu HY, Hua KF, Wu WC, Hsu J, Weng ST, Lin TL, Liu CY, et al. Reishi immuno-modulation protein induces interleukin-2 expression via protein kinase-dependent signaling pathways within human T cells. J Cell Physiol. 2008; 215:15-26. 